\title{
AZUMAYA ALGEBRAS WITH GENERAL GRADINGS AND THEIR AUTOMORPHISMS*
}

\author{
By \\ Hisatoshi IKAI
}

\begin{abstract}
Some basic properties of graded Azumaya algebras are given systematically. Here, the grading is indexed by an arbitrary additive group and the notion of a graded Azumaya algebra is relative to a choice of a commutation factor. We also establish two exact sequences which generalize the Skolem-Noether theorem.
\end{abstract}

\section{Introduction}

Let $R$ be a commutative ring and $\Delta$ be an additive group. We will work in the category of unitary associative graded $R$-algebras of type $\Delta$. A graded Azumaya algebra, introduced by L. N. Childs, G. Garfinkel, and M. Orzech [6], is a special object in this category. This notion is a generalization of the finitedimensional central simple algebras, and the Brauer group of such graded algebras have been extensively studied by F. W. Long [7] and by M. Orzech [9]. In this article, we propose to give some basic properties of such algebras systematically, and to prove the Skolem-Noether theorem in a form of two exact sequences.

To be more precise, we choose a bimultiplicative map $\varepsilon$ on $\Delta \times \Delta$ with value in the unit group $R^{*}$ of $R$. As in [6], the notion of graded Azumaya algebras is defined relative to $\varepsilon$. Though any other confusion would not actually occurs, we have employed the prefix " $\varepsilon$-" whenever the map $\varepsilon$ is involved. Thus, our " $\varepsilon$ Azumaya" is a synonym of "graded Azumaya" in [6]. As in the ungraded case, the property " $\varepsilon$-Azumaya" admits various equivalent descriptions and this fact itself is one of the important theorems. Before stating our Skolem-Noether theorem, we review definitions in [6] together with additional generalities from Section one to Section four. In particular, we give in Section one a supplement to

\footnotetext{
*1991 Mathematics Subject Classification. Primary 16W50; Secondary 16H05, 15A66.

Received May 13, 1998.

Revised October 12, 1998.
} 
[6] which deal with generalities on graded invertible modules. Our SkolemNoether theorem is stated in Section five in a form of two exact sequences

$$
1 \longrightarrow R^{*} \longrightarrow A_{0}^{*} \stackrel{i_{A}^{0}}{\longrightarrow} \operatorname{Aut}(A) \stackrel{\psi_{A}^{\varepsilon}}{\longrightarrow} \operatorname{Picgr}^{\Delta}(R)
$$

and

$$
1 \longrightarrow R^{*} \longrightarrow \tilde{h} U(A) \stackrel{i_{A}^{\varepsilon}}{\longrightarrow} \operatorname{Aut}(A) \stackrel{\varphi_{A}^{\varepsilon}}{\longrightarrow} \operatorname{Pic}(R)
$$

Here, $A=\bigoplus_{\lambda \in \Delta} A_{\lambda}$ is an arbitrary $\varepsilon$-Azumaya algebra and the meanings of each members are as follows: Aut $(A)$ is the automorphism group of $A$ with respect to the structure of unitary graded algebra, and $i_{A}^{0}$ assigns to $u \in A_{0}^{*}$ the inner automorphism $x \mapsto u x u^{-1}$ of $A . \tilde{h} U(A)$ is the group of units $u$ in $A$ whose localization $u_{p}$ at any $p \in \operatorname{Spec} R$ is homogeneous, and the automorphism $i_{A}^{\varepsilon}(u)$ is characterized by the formula

$$
i_{A}^{\varepsilon}(u)\left(x_{v}\right):=\sum_{\mu \in \Delta} \varepsilon(v, \mu) u_{\mu} x_{v} u^{-1}
$$

for $v \in \Delta$ and $x_{v} \in A_{v}$. Pic $(R)$ is the ordinary Picard group of $\operatorname{Spec} R$ and $\operatorname{Picgr}^{\Delta}(R)$ is the group of graded invertible $R$-modules $L=\bigoplus_{\lambda \in \Delta} L_{\lambda}$ of type $\Delta$ modulo graded isomorphisms of degree zero (here the adjective invertible refers to $L$ as a ungraded $R$-module). $\psi_{A}^{\varepsilon}$ assigns to $\alpha \in \operatorname{Aut}(A)$ the class of $L^{\varepsilon}(\alpha)=\bigoplus_{\lambda \in \Delta} L^{\varepsilon}(\alpha)_{\lambda}$ where

$$
L^{\varepsilon}(\alpha)_{\lambda}:=\left\{x_{\lambda} \in A_{\lambda} \mid \alpha\left(a_{\mu}\right) x_{\lambda}=\varepsilon(\mu, \lambda) x_{\lambda} a_{\mu}, \mu \in \Delta, a_{\mu} \in A_{\mu}\right\} .
$$

It is an important consequence of $\varepsilon$-Azumaya that so constructed $L^{\varepsilon}(\alpha)$ is indeed invertible. Finally $\varphi_{A}^{\varepsilon}$ assigns to $\alpha \in \operatorname{Aut}(A)$ the class of the underlying ungraded $R$-module of $L^{\varepsilon}(\alpha)$.

When we take take $\Delta=0$ and $\varepsilon=1$, the $\varepsilon$-Azumaya algebras are the same as the ordinary Azumaya algebras, and both (1) and (2) reduce to the exact sequence

$$
1 \longrightarrow R^{*} \longrightarrow A^{*} \stackrel{\text { int. }}{\longrightarrow} \operatorname{Aut}(A) \longrightarrow \operatorname{Pic}(R)
$$

due to Rosenberg and Zelinsky (cf. [10], [2, III-4.6]). In particular if $R$ is a field, we are reduced to considering central simple algebras and (3) recovers a part of the Skolem-Noether theorem (cf. [4, VIII, $\S 10$, no 1]). On the other hand, the case where $\Delta=Z / 2 Z$ and $\varepsilon(\lambda, \mu)=(-1)^{\lambda \mu}$ is treated in the works of H. Bass [2] and of C. Small [11]. In fact, our work is modeled on [2] and [11]. Among the topics in there, we devoted our attention to various definitions of Azumaya, 
which is important in producing examples, and to exact sequences describing automorphism groups. Here we keep in mind a relation to the theory of quadratic forms. Indeed, one of the motivations to consider such algebras comes from the fact that an example is given by the Clifford algebra $C(P, q)$ of a non-singular quadratic form $q$ on a finitely generated projective $R$-module $P$ (cf. $[2, \mathrm{~V}-3.9])$. Also exact sequences (1) and (2) in this case are utilized to construct various groups related to the orthogonal group $O(P, q)$ (cf. $[3, \S 3]$ ).

Finally, we would like to thank the referee for valuable comments and teaching us the works [6], [7], and [9].

\section{§1. Graded Invertible Modules}

1. We start with a general observation on an adjoint situation, by which we understand a data $(\varphi ; F, G ; \mathscr{D}, \mathscr{C})$ consisting of two categories $\mathscr{C}$ and $\mathscr{D}$, two functors

$$
F: \mathscr{D} \rightarrow \mathscr{C} \text { and } \quad G: \mathscr{C} \rightarrow \mathscr{D}
$$

and a natural equivalence $\varphi$ of set-valued bifunctors $\mathscr{D}(G(\cdot), \cdot) \stackrel{\sim}{\rightarrow} \mathscr{C}(\cdot, F(\cdot))$ (cf. $[8$, pp. 117-118]). Here, $\mathscr{A}(x, y)$ stands for the set of morphisms in a category $\mathscr{A}$ with source $x$ and target $y$. By definition, we have a bijection

$$
\varphi(c, d): \mathscr{D}(G(c), d) \stackrel{\sim}{\rightarrow} \mathscr{C}(c, F(d))
$$

for each $c \in \mathscr{C}$ and $d \in \mathscr{D}$. Denote by $\xi: \operatorname{Id}_{\mathscr{C}} \rightarrow F \circ G$ and by $\eta: G \circ F \rightarrow \operatorname{Id}_{\mathscr{D}}$ the associating natural transformations of functions (cf. [8, Ch. V, Prop. 1.1]), in other words, we have

$$
\left\{\begin{array}{l}
\xi(c)=\varphi(c, G(c))\left(\operatorname{Id}_{G(c)}\right) \in \mathscr{C}(c, F \circ G(c)), \\
\eta(d)=\varphi(F(d), d)^{-1}\left(\operatorname{Id}_{F(d)}\right) \in \mathscr{D}(G \circ F(d), d),
\end{array}\right.
$$

for $c \in \mathscr{C}$ and $d \in \mathscr{D}$. Then, we have the following lemma by means of which we will define the "graded invertible modules" and the " $\varepsilon$-Azumaya algebras" (cf. 3, 31):

LEMMA. The following conditions are equivalent:

(i) $F$ is an equivalence.

(ii) $G$ is an equivalence.

(iii) All the morphisms $\xi(c): c \rightarrow F \circ G(c)$ and $\eta(d): G \circ F(d) \rightarrow d$, where $c \in \mathscr{C}$ and $d \in \mathscr{D}$, are isomorphisms. 
Proof. We have [(i) or (ii)] $\Rightarrow$ (iii) from [8, Ch. V, Prop. 1.3]. (iii) amounts to saying that the natural transformations $\xi: \operatorname{Id}_{\mathscr{C}} \rightarrow F \circ G$ and $\eta: G \circ F \rightarrow \operatorname{Id}_{\mathscr{D}}$ are equivalences. Hence we have (iii) $\Rightarrow[($ i) and (ii)] from [8, Ch. II, Prop. 10.1].

2. Let $\Delta$ be an additive monoid such that any element $\lambda$ is regular (i.e., the translation $\delta \mapsto \delta+\lambda$ is injective). Let $A=\bigoplus_{\lambda \in \Delta} A_{\lambda}$ and $B=\bigoplus_{\lambda \in \Delta} B_{\lambda}$ be two graded rings of type $\Delta$, and $U=\bigoplus_{\lambda \in \Delta} U_{\lambda}$ be a graded $(B ; A)$-bimodule of type $\Delta$. Here and in sequel, we follow to $[4$, II, $\S 11]$ and $[4$, III, §4] the definitions and notations for graded rings, modules, and algebras. Denote by $C$-Mod, for $C=A$ and $B$, the category of graded left $C$-modules of type $\Delta$ with morphisms graded $C$-homomorphisms of degree zero (cf. [4, II, §11, def. 4]). By [4, II, §11, nos 5, 6], we then have an adjoint situation (cf. 1)

$$
\left(\varphi^{\mathrm{gr}} ; \operatorname{Homgr}_{B}(U, \cdot), U \oplus_{A} ; B \text {-Mod, } A \text {-Mod }\right),
$$

where $\varphi^{\mathrm{gr}}$ assigns to $M \in A$-Mod and $N \in B$-Mod the isomorphism $\varphi^{\mathrm{gr}}(M, N)$ : $B-\operatorname{Mod}\left(U \otimes_{A} M, N\right) \stackrel{\sim}{\rightarrow} A-\operatorname{Mod}\left(M, \operatorname{Homgr}_{B}(U, N)\right)$ of abelian groups sending $g: U \otimes_{A} M \rightarrow N$ to the map $g^{\prime}: M \rightarrow \operatorname{Homgr}_{B}(U, N)$ such that $g^{\prime}(m)(u):=$ $g(u \otimes m)$. As in 1 , denote by $\xi^{\mathrm{gr}}$ and by $\eta^{\mathrm{gr}}$ the natural transformations associating to the situation (1). By definition, $\xi^{\mathrm{gr}}$ and $\eta^{\mathrm{gr}}$ assign to $M \in A-\mathbf{M o d}$ and $N \in B$-Mod the homomorphisms

$$
\xi_{M}^{\mathrm{gr}}: M \rightarrow \operatorname{Homgr}_{B}\left(U, U \otimes_{A} M\right) \text { and } \eta_{N}^{\mathrm{gr}}: U \otimes_{A} \operatorname{Homgr}_{B}(U, N) \rightarrow N
$$

such that $\xi_{M}^{\mathrm{gr}}(m)(u)=u \otimes m$ and $\eta_{N}^{\mathrm{gr}}(u \otimes f)=f(u)$, respectively.

3. By the lemma in $\mathbf{1}$, the following definition is valid:

Definition. Let $(\Delta, A, B)$ be as in 2 and $U=\bigoplus_{\lambda \in \Delta} U_{\lambda}$ be a graded $(B ; A)$ bimodule of type $\Delta$. We say that $U$ is graded invertible if it satisfies the following conditions which are equivalent:

(i) The functor $U \otimes_{A}: A$-Mod $\rightarrow B$-Mod is an equivalence.

(ii) The functor $\operatorname{Homgr}_{B}(U, \cdot): B$-Mod $\rightarrow A$-Mod is an equivalence.

(iii) All the morphisms $\xi_{M}^{\mathrm{gr}}: M \rightarrow \operatorname{Homgr}_{B}\left(U, U \otimes_{A} M\right)$ and $\eta_{N}^{\mathrm{gr}}: U \otimes_{A}$ $\operatorname{Homgr}_{B}(U, N) \rightarrow N$, where $M \in A$-Mod and $N \in B$-Mod, are isomorphisms.

4. Let $A$ and $B$ be two ungraded rinds and $U$ be a ungraded $(B ; A)$ bimodule. When we apply 2 after taking $\Delta$ to be zero, the adjoint situation (1) of 2 reduces to the ordinary situation

$$
\left(\varphi ; \operatorname{Hom}_{B}(U, \cdot), U \otimes_{A} ; B \text {-mod } A \text {-mod }\right),
$$


where $C$-mod, for $C=A$ and $B$, is the category of ungraded left $C$-modules with morphisms $C$-homomorphisms, and $\varphi$ is the natural equivalence of bifunctors which assigns to $M \in A$-mod and $N \in B$-mod the canonical isomorphism $\varphi(M, N): \operatorname{Hom}_{B}\left(U \otimes_{A} M, N\right) \stackrel{\sim}{\rightarrow} \operatorname{Hom}_{A}\left(M, \operatorname{Hom}_{B}(U, N)\right)$ of abelian groups (cf. e.g. [4, II, §4. no 1]). Also we denote by

$$
\xi_{M}: M \rightarrow \operatorname{Hom}_{B}\left(U, U \otimes_{A} M\right) \text { and } \eta_{N}: U \otimes_{A} \operatorname{Hom}_{B}(U, N) \rightarrow N
$$

the associating homomorphisms. Now the graded invertibility of $U$, in the sense of 3 , reduces to the following equivalent conditions:

(i) $U \otimes_{A}: A$-mod $\rightarrow B$-mod is an equivalence.

(ii) $\operatorname{Hom}_{B}(U, \cdot): B$-mod $\rightarrow A$-mod is an equivalence.

(iii) All the morphisms $\xi_{M}$ and $\eta_{N}$ are isomorphisms.

The condition (i) is precisely the one for $U$ being invertible in the sense of $[2$, II-3.2]. Recall (cf. [2, II-5.6 (c), II-4.4 (1)]) that the latter is equivalent to the following equivalent conditions:

(iv) $U$ is faithfully projective (i.e., finitely generated, projective, and a generator) as left $B$-module, and the canonical ring homomorphism $A^{o} \rightarrow \operatorname{End}_{B}(U)$ is an isomorphism.

(v) $U$ is faithfully projective as right $A$-module, and the canonical ring homomorphism $B \rightarrow \operatorname{End}_{A}(U)$ is an isomorphism.

Recall also (cf. [2, II-4.3 (3), 5.6 (b), 5.10]) that, in the case where $A$ is commutative, these are equivalent to both of the following:

(vi) $U$ is a generator as left $B$-module, and the canonical ring homomorphism $A \rightarrow \operatorname{End}_{B}(U)$ is an isomorphism.

(vii) $U$ is finitely generated, projective, and faithful as $A$-module, and the canonical ring homomorphism $B \rightarrow \operatorname{End}_{A}(U)$ is an isomorphism.

5. THEOREM. Let $(\Delta, A, B)$ be as in $\mathbf{2}$ and $U=\bigoplus_{\lambda \in \Delta} U_{\lambda}$ be a graded $(B ; A)$ bimodule of type $\Delta$. Assume that $\Delta$ is a group. Then the following two conditions are equivalent:

(i) $U$ is graded invertible, in the sense of $\mathbf{3}$.

(ii) The underlying ungraded $(B ; A)$-bimodule of $U$ is invertible, in the sense of [2, II-3.2].

Proof. (i) $\Rightarrow$ (ii): We shall verify the condition (v) in 4 .

$1^{\circ}$ The canonical homomorphism $A^{o} \rightarrow \operatorname{Endgr}_{B}(U)$ of graded rings is an isomorphism.-To prove this, denote by $A(\delta)$ (resp. $U(\delta)$ ) the object in $A$-Mod (resp. $B$-Mod) obtained from $A$ (resp. $U$ ) by shifting the grading by $\delta \in \Delta$ (cf. [4, 
II, §11, no 2, Example (3)]). The scalar multiplications induce homomorphisms $e_{\delta}: A_{\delta} \rightarrow A-\operatorname{Mod}(A(0), A(\delta)) \quad$ and $\quad \psi_{\delta}: A_{\delta} \rightarrow B-\operatorname{Mod}(U(0), U(\delta)) \quad$ of abelian groups, and morphisms $\chi_{\delta}: U \otimes_{A} A_{\delta} \rightarrow U(\delta)$ in the category $B$-Mod. Note that all the $e_{\delta}$ 's and $\chi_{0}$ are isomorphisms, and that the diagram

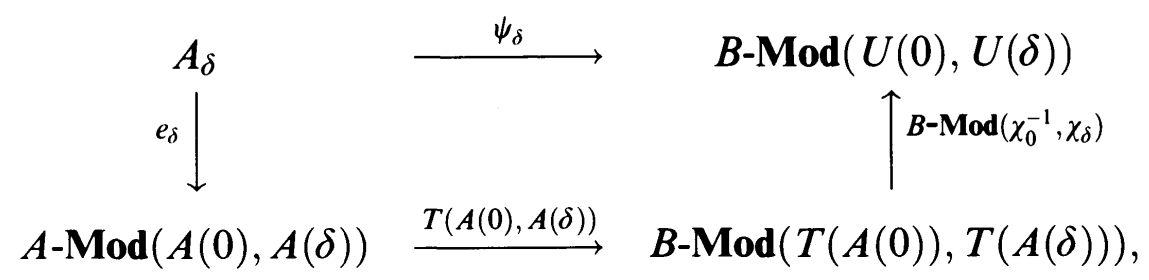

where $T$ stands for the functor $U \otimes_{A}$, is commutative. In our situation, all the $\chi_{\delta}$ 's are also isomorphism since $\Delta$ is a group, and so are $T(A(0), A(\delta))$ 's since $T=U \otimes_{A}$ is an equivalence (cf. 3). It follows that $\psi_{\delta}$ 's are all isomorphisms. Hence so is the map in question, which is precisely the direct sum $\bigoplus_{\delta \in \Delta} \psi_{\delta}$.

$2^{\circ}$ - The lemma in 6 below, combined with the surjectivity of the map $\eta_{N}^{\mathrm{gr}}$ for $N=B$ (cf. 3 (2)), tells us that the underlying ungraded left $B$-module structure $\mathscr{S}$ on $U$ is faithful, that the canonical ungraded left $\operatorname{Endgr}_{B}(U)$-module structure $\mathscr{S}^{\prime}$ on $U$ is finitely generated projective, and that the endmorphism ring for $\mathscr{S}^{\prime}$ is the same as the homothety ring for $\mathscr{S}$. In view of $1^{\circ}$, these results can be read as follows: The underlying ungraded right $A$-module of $U$, say $U_{A}$, is finitely generated projective and the canonical ring homomorphism $B \rightarrow \operatorname{End}_{A}(U)$ is an isomorphism.

$3^{\circ}$ - It remains to show that $U_{A}$ is a generator. $U_{A}$ may be considered to be dual to the ungraded right $A$-module $\left(U_{A}\right)^{*}:=\operatorname{Hom}_{A}\left(U_{A}, A\right)$, since $U_{A}$ is finitely generated and projective (cf. $\left.2^{\circ}\right)$. Hence it suffices to show that $\left(U_{A}\right)^{*}$ is a generator (cf. [2, II-5.6 (b)]), i.e. the functor $\operatorname{Hom}_{A}\left(\left(U_{A}\right)^{*}, \cdot\right.$ ) from $A$-mod to the category $\mathbf{A b}$ of abelian groups is faithful. Indeed, it is isomorphic to the composite $A$-mod $\stackrel{G}{\rightarrow} A$-Mod $\stackrel{T}{\rightarrow} B$-Mod $\stackrel{F}{\rightarrow}$ Ab, where $G$ is the functor giving the trivial grading which is faithful, $T$ is the functor $U \otimes_{A}$ which is an equivalence (cf. 3), and $F$ is the forgetful functor which is faithful.

(ii) $\Rightarrow$ (i): $\Delta$ is a group and the underlying ungraded left $B$-module of $U$ is finitely generated (cf. 4 (iv)). It follows that $\operatorname{Hom}_{B}(U, Y)$ is precisely the underlying ungraded left $A$-module of $\operatorname{Homgr}_{B}(U, Y)$, for any $Y$ in $B$-Mod (cf. [4, II, $\S 11$, no 6, Remark]). Hence, after forgetting the gradings, we have $\xi_{M}^{\mathrm{gr}}=\xi_{M}$ and $\eta_{N}^{\mathrm{gr}}=\eta_{N}$ for all $M \in A$-Mod and all $N \in B$-Mod. Since $\xi_{M}$ and $\eta_{N}$ are all isomorphisms (cf. 4 (iii)), so are $\xi_{M}^{\mathrm{gr}}$ and $\eta_{N}^{\mathrm{gr}}$.

6. Lemma. Let $\Delta$ be an additive monoid whose elements are regular, $A$ be a graded ring of type $\Delta$, and $E$ be a graded left A-module of type $\Delta$. Assume that 
there exist finite families of elements $x_{1}, \ldots, x_{n} \in E$ and $f_{1}, \ldots, f_{n} \in \operatorname{Homgr}_{A}(E, A)$ such that $\sum_{1 \leq i \leq n} f_{i}\left(x_{i}\right)=1$. Then

(i) The underlying ungraded left A-module structure on $E$ is faithful.

(ii) The canonical ungraded left $\operatorname{Endgr}_{A}(E)$-module structure on $E$ is finitely generated and projective.

(iii) Any endmorphism $v$ of $E$ with respect to the left $\operatorname{Endgr}_{A}(E)$-module structure in (ii) is a scalar multiplication by an element of $A$.

This lemma is proved in the same way as in [2, II-4.3].

7. Let $(\Delta, A, B)$ be as in 2. Then the group $\operatorname{Homgr}_{B}(U, B)$ carries a structure of right $B$-module as well as the structure of graded left $A$-module of type $\Delta$ defined in 2 . The former is defined by the formula $(f b)(u):=f(u) b$ for $b \in B, f \in \operatorname{Homgr}_{B}(U, B)$ and $u \in U$. By these structures, we regard $\operatorname{Homgr}_{B}(U, B)$ as a graded $(A ; B)$-bimodule of type $\Delta$. Then, by applying 2 after interchanging $A$ and $B$, we obtain a functor $\operatorname{Homgr}_{B}(U, B) \otimes_{B}$ from $B$-Mod to $A$ Mod. Also we have a morphism

$$
\theta_{N}^{\mathrm{gr}}: \operatorname{Homgr}_{B}(U, B) \otimes_{B} N \rightarrow \operatorname{Homgr}_{B}(U, N)
$$

in $A$-Mod, for each $N \in B$-Mod functorially, such that $\theta_{N}^{\mathrm{gr}}(f \otimes n)(u)=f(u) \cdot u$.

Corollary. Let $(\Delta, A, B, U)$ be as in 2 , and assume that $\Delta$ is a group and that $U$ is graded invertible. Then the graded bimodule $\operatorname{Homgr}_{B}(U, N)$ is also graded invertible, and there exist isomorphisms

$$
U \otimes_{A} \operatorname{Homgr}_{B}(U, B) \stackrel{\sim}{\rightarrow} B \text { and } \operatorname{Homgr}_{B}(U, B) \otimes_{B} U \stackrel{\sim}{\rightarrow} A
$$

of graded $(B ; B)$-bimodules and of graded $(A ; A)$-bimodules, respectively.

ProOF. Since the graded invertibility reduces to the invertibility of underlying ungraded bimodule (cf. 5), it suffices to establish isomorphisms (2) (cf. [2, II-3.2]). Indeed, the former is furnished by the natural isomorphism $\eta_{N}^{\mathrm{gr}}$ (cf. 3) for $N=B$. On the other hand, note that $\Delta$ is a group and that $U$ is finitely generated and projective as ungraded left $B$-module (cf. 5, 4 (iv)). It follows that we have $\operatorname{Homgr}_{B}(U, N)=\operatorname{Hom}_{B}(U, N)$ after forgetting grading (cf. [4, II, §11, no 6, Remark]). Hence the morphism $\theta_{N}^{\mathrm{gr}}$ (cf. (1)) reduces to the canonical homomorphism $\theta_{N}: \operatorname{Hom}_{B}(U, B) \otimes_{B} N \rightarrow \operatorname{Hom}_{B}(U, N)$ which is an isomorphism (cf. [4, $\S 4$, no 2, Prop. 2, Cor.]). Therefore, $\theta_{N}^{\mathrm{gr}}$ is an isomorphism, and so is $\Phi: A^{o} \rightarrow \operatorname{Endgr}_{B}(U)$, the canonical homomorphism of graded rings, by the same 
argument. Thus the latter of (2) is furnished by the composite of $\theta_{N}^{\mathrm{gr}}$ for $N=U$ with $\Phi^{-1}$.

8. Let $\Delta$ be an additive group and $A=\bigoplus_{\lambda \in \Delta} A_{\lambda}$ be a graded ring of type $\Delta$. If $P$ and $Q$ are graded $(A ; A)$-bimodules of type $\Delta$, the tensor product $P \otimes_{A} Q$ acquires also a canonical structure of such graded bimodule. In this way, we regard $\otimes_{A}$ as a product in the category $A$-Mod- $A$ of graded $(A ; A)$-bimodules of type $\Delta$. This product is associative up to natural isomorphisms, while it is not commutative in general. Also, for $P \in A$-Mod- $A$, we put

$$
\check{P}:=\operatorname{Homgr}_{A}\left({ }_{A} P, A\right),
$$

where ${ }_{A} P$ stands for the underlying graded left $A$-module of $P$, to obtain an object $\check{P}$ in $A$-Mod- $A$ (cf. 7). In this way, we regard $\check{?}$ as an operator in the category $A$-Mod- $A$. Since the composite functor $A$-Mod $\stackrel{P \otimes_{A}}{\longrightarrow} A$-Mod $\stackrel{Q \otimes_{A}}{\longrightarrow} A$-Mod is isomorphic to $\left(P \otimes_{A} Q\right) \otimes_{A}$, we see that, in the category $A$-Mod- $A$, the graded invertible bimodules are closed under the product $\otimes_{A}$. Moreover by 7 , if $P \in$ $A$-Mod- $A$ is graded invertible, so is $\check{P}$ and we have $P \otimes_{A} \check{P} \stackrel{\sim}{\rightarrow} \check{P} \otimes_{A} P \stackrel{\sim}{\rightarrow} A$. Thus, by the product induced by $\otimes_{A}$, the isomorphism classes $\operatorname{cl}(P)$ with $P$ graded invertible form a group in which we have $\operatorname{cl}(P)^{-1}=\operatorname{cl}(\breve{P})$. We call this group the graded Picard group of $A$, and denote it by $\operatorname{Picgr}(A)$.

9. Let $\Delta$ be an additive group and $A=\bigoplus_{\lambda \in \Delta} A_{\lambda}$ be a graded ring of type $\Delta$. We denote by $\operatorname{Aut}(A)$ the automorphism group of $A$ with respect to the graded ring structure. For $\alpha, \beta \in \operatorname{Aut}(A)$ and $P \in A$-Mod- $A$, denote by ${ }_{\alpha} P_{\beta}$ the object in $A$-Mod- $A$ having the same underlying graded abelian group as $P$, and with multiplication $a \cdot x \cdot b:=\alpha(a) \times \beta(b)$ for $a, b \in A$ and $x \in P$. We use the notation ${ }_{\alpha}|x|_{\beta}$ to denote the element of ${ }_{\alpha} P_{\beta}$ which is identified with an element $x \in P$ in the underlying abelian group. Hence, we have $a_{\alpha}|x|_{\beta} b={ }_{\alpha}|\alpha(a) x \beta(b)|_{\beta}$. If $\gamma \in \operatorname{Aut}(A)$ and $Q \in A$-Mod- $A$, we have an isomorphism ${ }_{\alpha} P_{\beta} \otimes_{A} Q_{\gamma} \stackrel{\sim}{\rightarrow}{ }_{\alpha}\left(P \otimes_{A} Q\right)_{\gamma}$ given by the map sending ${ }_{\alpha}|x|_{\beta} \otimes_{\beta}|y|_{\gamma}$ to ${ }_{\alpha}|x \otimes y|_{\gamma}$. Taking $P$ to be $A$, we obtain an object ${ }_{\alpha} A_{\beta}$ in $A$-Mod- $A$. In this case, an isomorphism ${ }_{\alpha} A_{\beta} \stackrel{\sim}{\rightarrow}{ }_{\gamma \alpha} A_{\gamma \beta}$ is given by the map sending ${ }_{\alpha}|x|_{\beta}$ to ${ }_{\gamma \alpha}|\gamma(x)|_{\gamma \beta}$. Thus, we get an isomorphism.

$$
{ }_{1} A_{\alpha} \otimes_{A 1} A_{\beta} \stackrel{\sim}{\rightarrow}{ }_{1} A_{\alpha \beta}
$$

given by the map sending ${ }_{1}|x|_{\alpha} \otimes_{1}|y|_{\beta}$ to ${ }_{1}|x \alpha(y)|_{\alpha \beta}$, which is the composite ${ }_{1} A_{\alpha} \otimes_{A 1} A_{\beta} \stackrel{\sim}{\rightarrow}{ }_{1} A_{\alpha} \otimes_{A \alpha} A_{\alpha \beta} \stackrel{\sim}{\rightarrow}{ }_{1}\left(A \otimes_{A} A\right)_{\alpha \beta} \stackrel{\sim}{\rightarrow}{ }_{1} A_{\alpha \beta}$ of the above isomorphisms. (1) tells us that ${ }_{1} A_{\alpha}$ is graded invertible (cf. 5, 4 (i)) and that the map 
$\varphi_{A}: \operatorname{Aut}(A) \rightarrow \operatorname{Picgr}(A)$ given by

$$
\varphi_{A}(\alpha):=\operatorname{cl}\left({ }_{1} A_{\alpha}\right) \in \operatorname{Picgr}(A)
$$

is a homomorphism of groups. On the other hand, recall that the zero-component $A_{0}$ is a subring of $A$ and that the center $\operatorname{Cen}(A)$ of the underlying ungraded ring of $A$ is a graded subring of $A$ (cf. [4, $\S 11$, no 2, Prop. 1, no 3, Prop. 5, Cor.]). Denote by $A_{0}^{*}$ the multiplicative group of units is $A_{0}$, and by $i_{A}^{0}(u)$ the inner automorphism of $A$ induced by an element $u$ of $A_{0}^{*}$. Then $i_{A}^{0}$ may be considered as a homomorphism $A_{0}^{*} \rightarrow \operatorname{Aut}(A)$ of groups. Note that $A_{0}^{*} \cap \operatorname{Cen}(A)$, which is the kernel of $i_{A}^{0}$, is in fact the multiplicative group $\operatorname{Cen}(A)_{0}^{*}$ of the zero-component of $\operatorname{Cen}(A)_{0}$. The following proposition is proved in the same way as in [2, II-7.2]:

Proposition. The sequence

$$
1 \longrightarrow \operatorname{Cen}(A)_{0}^{*} \longrightarrow A_{0}^{*} \stackrel{i_{A}^{0}}{\longrightarrow} \operatorname{Aut}(A) \stackrel{\varphi_{A}}{\longrightarrow} \operatorname{Picgr}(A)
$$

is exact and the image of $\varphi_{A}$ consists of $\mathrm{cl}(P)^{\prime}$ 's with $P \in A$-Mod- $A$ graded invertible whose underlying graded left $A$-module is isomorphic to that of $A$.

\section{§ 2. Generalities on Graded Algebras}

10. Let $R$ be a commutative ring and $\Delta$ be an additive monoid whose elements are all regular. We begin with recalling the notions of commutation factors and of graded tensor $\varepsilon$-products from [4, III, $\S 4$, nos 6-9]. By a commutation factor over $\Delta$ with value in $R$, we understand a map $\varepsilon: \Delta \times \Delta \rightarrow R$ satisfying $\varepsilon\left(\lambda, \mu+\mu^{\prime}\right)=\varepsilon(\lambda, \mu) \varepsilon\left(\lambda, \mu^{\prime}\right), \varepsilon\left(\lambda+\lambda^{\prime}, \mu\right)=\varepsilon(\lambda, \mu) \varepsilon\left(\lambda^{\prime}, \mu\right)$, and $\varepsilon(\lambda, \mu) \varepsilon(\mu$, $\lambda)=1$. By definition, the map $(\lambda, \mu) \mapsto \varepsilon(\mu, \lambda)$ is also a commutation factor which we denote by $\check{\varepsilon}$. If $A=\bigoplus_{\lambda \in \Delta} A_{\lambda}$ and $B=\bigoplus_{\lambda \in \Delta} B_{\lambda}$ are two graded $R$-algebras of type $\Delta$, we have a similar algebra $A \otimes_{R}^{\varepsilon} B$ called graded tensor $\varepsilon$-product of type $\Delta$ (cf. [4, III, $\S 3$, no 8]). By definition, the underlying graded $R$-module of $A \otimes_{R}^{\varepsilon} B$ is the ordinary tensor product $A \otimes_{R} B$ with the total grading, while the multiplication is such that

$$
\left(a \otimes b_{\mu}\right)\left(x_{\lambda} \otimes y\right)=\varepsilon(\mu, \lambda)\left(a x_{\lambda}\right) \otimes\left(b_{\mu} y\right)
$$

for $a \in A, y \in B, \lambda, \mu \in \Delta, x_{\lambda} \in A_{\lambda}$, and $b_{\mu} \in B_{\mu}$ (cf. [4, III, $\S 4$, no 7, (21)]. Thus, for any graded $R$-algebra $F$ of type $\Delta$ and any diagram $A \stackrel{f}{\rightarrow} F \stackrel{g}{\leftarrow} B$ with $g\left(b_{\mu}\right) f\left(a_{\lambda}\right)=\varepsilon(\mu, \lambda) f\left(a_{\lambda}\right) g\left(b_{\mu}\right)$ for $\lambda, \mu \in \Delta, a_{\lambda} \in A_{\lambda}$ and $b_{\mu} \in B_{\mu}$, the $R$-linear map $h: A \otimes_{R}^{\varepsilon} B \rightarrow F$ sending $a \otimes b$ to $f(a) g(b)$ is also a homomorphism of graded 
algebras. If $S$ is another commutative ring and $\varphi: R \rightarrow S$ is a ring homomorphism, we denote by $\varphi \varepsilon$ the composite

$$
\varphi \varepsilon: \Delta \times \Delta \stackrel{\varepsilon}{\rightarrow} R \stackrel{\varphi}{\rightarrow} S
$$

which is clearly a commutation factor over $\Delta$ with value in $S$. Also we denote by $\varphi^{*} A$ the graded $S$-algebra of type $\Delta$ derived from a graded $R$-algebra $A$ by the scalar extension relative to $\varphi$. Observe that the composite isomorphism $\varphi^{*}\left(A \otimes_{R}^{\varepsilon} B\right) \stackrel{\sim}{\rightarrow} S \otimes_{R}\left(A \otimes_{R} B\right) \stackrel{\sim}{\rightarrow}\left(S \otimes_{R} A\right) \otimes_{S}\left(S \otimes_{R} B\right) \stackrel{\sim}{\rightarrow}\left(\varphi^{*} A\right) \otimes_{S}^{\varphi \varepsilon}\left(\varphi^{*} B\right)$ of underlying ungraded $S$-modules gives rise to an isomorphism $\varphi^{*}\left(A \otimes_{R}^{\varepsilon} B\right) \stackrel{\sim}{\rightarrow}$ $\left(\varphi^{*} A\right) \otimes_{S}^{\varphi \varepsilon}\left(\varphi^{*} B\right)$ of graded $S$-algebras. We say that this isomorphism is canonical.

Let us introduce some more notations. We denote by $A^{\varepsilon}$ the similar algebra having the same underlying graded $R$-module as $A$, and with multiplication $a_{\lambda} \cdot b_{\mu}:=\varepsilon(\lambda, \mu) a_{\lambda} b_{\mu}$ for $\lambda, \mu \in \Delta, a_{\lambda} \in A_{\lambda}$ and $b_{\mu} \in A_{\mu}$. We use the notation $a^{\varepsilon}$ to denote the element of $A^{\varepsilon}$ which is identified with an element $a \in A$ in the underlying module. In this notation, we have $a_{\lambda}^{\varepsilon} b_{\mu}^{\varepsilon}=\varepsilon(\lambda, \mu)\left(a_{\lambda} b_{\mu}\right)^{\varepsilon}$. Also we observe $A^{\varepsilon \check{\varepsilon}}=A^{\check{\varepsilon} \varepsilon}=A$ and $A^{o \varepsilon}=A^{\check{\varepsilon} o}$, where $A^{o}$ denotes the opposite algebra of $A$ in general. We use the similar notation $a^{o \varepsilon} \in A^{o \varepsilon}$ for $a \in A$ as above. Note that we then have $a_{\lambda}^{o \varepsilon} b_{\mu}^{o \varepsilon}=\varepsilon(\lambda, \mu)\left(b_{\mu} a_{\lambda}\right)^{o \varepsilon}$.

11. Let $(R, \Delta, \varepsilon)$ be as in 10. In sequel, we use the following conventions: For any graded $R$-algebra $A=\bigoplus_{\lambda \in \Delta} A_{\lambda}$ of type $\Delta$, we denote by $A$-Mod (resp. Mod- $A$ ) the category of graded left (resp. right) $A$-modules of type $\Delta$ with morphisms $A$-homomorphisms of degree zero. Let $B=\bigoplus_{\lambda \in \Delta} B_{\lambda}$ be another graded $R$-algebra. By a statement " $M$ is a graded $(A ; B)$-bimodule of type $\Delta$ ", we understand that both $A$ and $B$ induce the same $R$-module structure on $M$. Denote by $A$-Mod- $B$ the category of graded $(A ; B)$-bimodule of type $\Delta$ in this sense with morphisms $(A ; B)$-homomorphisms of degree zero.

These being said, consider the algebra $A \otimes_{R}^{\varepsilon} B^{o \varepsilon}$, in which we have the multiplication formula $\left(a \otimes b_{\mu}^{o \varepsilon}\right)\left(x_{v} \otimes y_{\sigma}^{o \varepsilon}\right)=\varepsilon(\mu, v+\sigma)\left(a x_{v}\right) \otimes\left(y_{\sigma} b_{\mu}\right)^{o \varepsilon}$ for $a \in A$, $\mu, v, \sigma \in \Delta, b_{\mu} \in A_{\mu}, x_{v} \in A_{v}$ and $y_{\sigma} \in B_{\sigma}$. Then we have an identification

$$
A \text {-Mod- } B \simeq\left(A \otimes_{R}^{\varepsilon} B^{o \varepsilon}\right)-\operatorname{Mod}
$$

of categories as follows: If $M=\bigoplus_{\lambda \in \Delta} M_{\lambda}$ is in $A$-Mod- $B$, we regard $M$ as an object in $\left(A \otimes_{R}^{\varepsilon} B^{o \varepsilon}\right)$-Mod by preserving the underlying graded $R$-module and by the multiplication formula

$$
\left(a \otimes b_{\mu}^{o \varepsilon}\right) \cdot x_{v}:=\varepsilon(\mu, v) a x_{v} b_{\mu} .
$$

Conversely if $N=\bigoplus_{\lambda \in \Delta} N_{\lambda}$ is in $\left(A \otimes_{R}^{\varepsilon} B^{o \varepsilon}\right)$-Mod, we regard $N$ as an object in 
$A$-Mod- $B$, by preserving the underlying graded $R$-module and by the multiplication formulas

$$
a \cdot x:=\left(a \otimes 1^{o \varepsilon}\right) x \quad \text { and } \quad x_{v} \cdot b_{\mu}:=\varepsilon(v, \mu)\left(1 \otimes b_{\mu}^{o \varepsilon}\right) x_{v} .
$$

In either case morphisms need not be changed. It is a simple calculation to see that (2) and (3) indeed define required module structures and that so constructed two functors are inverses each other. We observe also that the identification (1) commutes with base change in the obvious sense.

12. Let $(R, \Delta, \varepsilon)$ be as in $\mathbf{1 0}$ and $A=\bigoplus_{\lambda \in \Delta} A_{\lambda}$ be a graded $R$-algebra of type $\Delta$. We then define a similar algebra $A_{\varepsilon}^{e}$ by

$$
A_{\varepsilon}^{e}:=A \otimes_{R}^{\varepsilon} A^{o \varepsilon},
$$

and call it the graded $\varepsilon$-enveloping algebra of $A$. Thus, $A_{\varepsilon}^{e}$ satisfies the multiplication formula $\left(a \otimes x_{\lambda}^{o \varepsilon}\right)\left(b_{\mu} \otimes y_{v}^{o \varepsilon}\right)=\varepsilon(\lambda, \mu+v)\left(a b_{\mu}\right) \otimes\left(y_{v} x_{\lambda}\right)^{o \varepsilon}$ for $a \in A, \lambda, \mu$, $v \in \Delta, \quad x_{\lambda} \in A_{\lambda}, b_{\mu} \in A_{\mu}$ and $y_{v} \in A_{\nu}$. Since $A$ has a graded $(A ; A)$-bimodule structure by the ring multiplication, it also has an induced graded left $A_{\varepsilon}^{e}$-module structure which is characterized by the formula $\left(a \otimes b_{\mu}^{o \varepsilon}\right) \cdot c_{v}=\varepsilon(\mu, v) a c_{v} b_{\mu}$ (cf. 11 (2)). The corresponding homomorphism

$$
\eta_{A}: A_{\varepsilon}^{e} \rightarrow \operatorname{Endgr}_{R}(A)
$$

is called the canonical representation of $A_{\varepsilon}^{e}$ in $A$. We have an $R$-linear map $\phi_{A}: A_{\varepsilon}^{e} \rightarrow A$ defined by $\phi_{A}\left(a \otimes x^{o \varepsilon}\right):=a x$, which is surjective and compatible with gradings. Moreover, viewing $A$ as a left $A_{\varepsilon}^{e}$-module by the canonical representation, we see that $\phi_{A}$ is also $A_{\varepsilon}^{e}$-linear. In this way, we obtain an exact sequence

$$
0 \longrightarrow \operatorname{ker} \phi_{A} \longrightarrow A_{\varepsilon}^{e} \stackrel{\phi_{A}}{\longrightarrow} A \longrightarrow 0
$$

of left $A_{\varepsilon}^{e}$-modules. In particular, $A$ is finitely generated as a left $A_{\varepsilon}^{e}$-module. On the other hand, the same calculation as in [2, III-1.2] shows that $\operatorname{ker} \phi_{A}$ is generated by $\left\{a \otimes 1^{o \varepsilon}-1 \otimes a^{o \varepsilon} \mid a \in A\right\}$ as a left ideal. The formation $A \mapsto A_{\varepsilon}^{e}$ of $\varepsilon$-enveloping algebras can be considered as a functor from the category of graded $R$-algebras of type $\Delta$ to itself. If $f: A \rightarrow B$ is a morphism of such algebras, $f_{\varepsilon}^{e}: A_{\varepsilon}^{e} \rightarrow B_{\varepsilon}^{e}$ is the map such that $f_{\varepsilon}^{e}\left(a \otimes x^{o \varepsilon}\right)=f(a) \otimes f(x)^{o \varepsilon}$. If we regard $A$ (resp. $B$ ) as a left module over $A_{\varepsilon}^{e}$ (resp. $B_{\varepsilon}^{e}$ ) by the canonical representation, then the map $f: A \rightarrow B$ becomes semi-linear relative to $f_{\varepsilon}^{e}: A_{\varepsilon}^{e} \rightarrow B_{\varepsilon}^{e}$, and we have $\phi_{B} f_{\varepsilon}^{e}=f \phi_{A}$. The formation of $\varepsilon$-enveloping algebras commutes with tensor $\varepsilon$ products $\otimes_{R}^{\varepsilon}$ in the sense that an isomorphism $\Phi_{A, B}: A_{\varepsilon}^{e} \otimes_{R}^{\varepsilon} B_{\varepsilon}^{e} \stackrel{\sim}{\rightarrow}\left(A \otimes_{R}^{\varepsilon} B\right)_{\varepsilon}^{e}$ can 
be defined by the formula $\Phi_{A, B}\left(\left(a \otimes x_{\mu}^{o \varepsilon}\right) \otimes\left(b_{v} \otimes y^{o \varepsilon}\right)\right)=\varepsilon(\mu, v)\left(a \otimes b_{v}\right) \otimes\left(x_{\mu} \otimes\right.$ $y)^{o \varepsilon}$. Our formation commutes also with base change in the obvious sense.

13. If $M=\bigoplus_{\lambda \in \Delta} M_{\lambda}$ is in $A$-Mod- $A$, we denote by $M_{\varepsilon}^{A}=\bigoplus_{\lambda \in \Delta}\left(M_{\varepsilon}^{A}\right)_{\lambda}$ the graded submodule of the underlying graded $R$-module of $M$ such that

$$
\left(M_{\varepsilon}^{A}\right)_{\lambda}:=\left\{x_{\lambda} \in M_{\lambda} \mid a_{\mu} x_{\lambda}=\varepsilon(\mu, \lambda) x_{\lambda} a_{\mu}, \forall_{\mu} \in \Delta, \forall a_{\mu} \in A_{\mu}\right\} .
$$

Observe that any morphism $u: M \rightarrow M^{\prime}$ in $A$-Mod- $A$ sends $M_{\varepsilon}^{A}$ into $M_{\varepsilon}^{\prime A}$. Denote by $u_{\varepsilon}^{A}$ the morphism $M_{\varepsilon}^{A} \rightarrow M_{\varepsilon}^{\prime A}$ in $R$-Mod induced by $u$. In this way, we obtain a functor

$$
M \mapsto M_{\varepsilon}^{A}: A \text {-Mod- } A \rightarrow R \text {-Mod }
$$

Recall from 11 and from 2 that we have an identification $A$-Mod- $A \stackrel{\sim}{\rightarrow} A_{\varepsilon}^{e}$-Mod and a functor $\operatorname{Homgr}_{A_{\varepsilon}^{e}}(A, \cdot)$ on $A_{\varepsilon}^{e}$-Mod, respectively. We propose to show that the functors (2) and $\operatorname{Homgr}_{A_{\varepsilon}^{e}}(A, \cdot)$ are isomorphic. Note first that, in terms of the $A_{\varepsilon}^{e}$-module structure, the definition of $M_{\varepsilon}^{A}$ can be read as

$$
M_{\varepsilon}^{A}=\left\{x \in M \mid z \cdot x=0, \forall z \in \operatorname{ker} \phi_{A}\right\} .
$$

This follows from the equation $a_{\mu} x_{\lambda}-\varepsilon(\mu, \lambda) x_{\lambda} a_{\mu}=\left(a_{\mu} \otimes 1^{o \varepsilon}-1 \otimes a_{\mu}^{o \varepsilon}\right) \cdot x_{\lambda}$ (cf. 11 (2)), and from the fact that $\operatorname{ker} \phi_{A}$ is a graded left ideal of $A_{\varepsilon}^{e}$ generated by homogeneous elements $a_{\mu} \otimes 1^{o \varepsilon}-1 \otimes a_{\mu}^{o \varepsilon}$ (cf. 12). The expression (3) makes it clear that any $A_{\varepsilon}^{e}$-linear map $f: A \rightarrow M$ carries 1 into $M_{\varepsilon}^{A}$. Note that we have $f(1) \in\left(M_{\varepsilon}^{A}\right)_{\lambda}$ if $f$ is graded of degree $\lambda$. Conversely, for any element $x_{\lambda}$ in $\left(M_{\varepsilon}^{A}\right)_{\lambda}$, the formula $f(a):=a x_{\lambda}$ defines an $A_{\varepsilon}^{e}$-linear map $f: A \rightarrow M$ of degree $\lambda$. It follows that the map $f \mapsto f(1)$ induces an isomorphism

$$
f \mapsto f(1): \operatorname{Homgr}_{A_{\varepsilon}^{e}}(A, M) \stackrel{\sim}{\rightarrow} M_{\varepsilon}^{A}
$$

in $R$-Mod. This isomorphism is functional in $M$ by construction. Hence, varying $M$, we obtain the announced isomorphism of functors.

Note also that the isomorphism (4), viewed as a $R$-linear map of ungraded $R$ modules, is in fact the composite $\operatorname{Homgr}_{A_{\varepsilon}^{e}}(A, M) \stackrel{\text { incl. }}{\longrightarrow} \operatorname{Hom}_{A_{\varepsilon}^{e}}(A, M) \stackrel{\Phi}{\rightarrow} M_{\varepsilon}^{A}$, where $\Phi(g):=g(1) . \Phi$ is injective since 1 generates $A$ as $A_{\varepsilon}^{e}$-module. Hence both of two maps in the above composite are bijective. Therefore, we have

$$
\operatorname{Homgr}_{A_{\varepsilon}^{e}}(A, M)=\operatorname{Hom}_{A_{\varepsilon}^{e}}(A, M) \text { as ungraded } R \text {-modules. }
$$

14. We consider the base change for the situation of 13. Let $S$ be another commutative ring and $\varphi: R \rightarrow S$ be a ring homomorphism. If $M=\bigoplus_{\lambda \in \Delta} M_{\lambda}$ is 
in $A$-Mod- $A$, it is clear from the definition 13 (1) that the natural morphism $M \rightarrow \varphi_{*} \varphi^{*} M$ induces, by restricting to $M_{\varepsilon}^{A}$ and extending scalars, a morphism

$$
H_{\varphi}(M): \varphi^{*}\left(M_{\varepsilon}^{A}\right) \rightarrow\left(\varphi^{*} M\right)_{\varphi \varepsilon}^{\varphi^{*} A}
$$

in $S$-Mod. Observe that, after forgetting gradings, we may use the isomorphisms (4) and (5) in 13 to identify $H_{\varphi}(M)$ with the $S$-linear map $\varphi^{*} \operatorname{Hom}_{A_{\varepsilon}^{e}}(A, M) \rightarrow$ $\operatorname{Hom}_{\varphi^{*}\left(A_{\varepsilon}^{e}\right)}\left(\varphi^{*} A, \varphi^{*} M\right)$ induced by the functoriality of $\varphi^{*}$. The following proposition is proved in the same way as in $[2$, II-2.2, 2.8]:

Proposition. Let $(R, \Delta, \varepsilon, A)$ be as in 13 , and $\varphi: R \rightarrow S$ be a homomorphism of commutative rings. Assume that the underlying ungraded $R$-module of $A$ is finitely presented, and that $S$ is flat over $R$. Then the morphism $H_{\varphi}(M)$ is an isomorphism for all $M \in A$-Mod- $A$.

15. Consider the graded $R$-module $A_{\varepsilon}^{A}$ of type $\Delta$ (cf. 13), and observe that it is in fact a subalgebra of $A$. We denote by

$$
\operatorname{Cen}^{\varepsilon}(A)=\bigoplus_{\lambda \in \Delta} \operatorname{Cen}^{\varepsilon}(A)_{\lambda}
$$

so constructed graded $R$-algebra of type $\Delta$, and call it the graded $\varepsilon$-center of $A$. Note that the isomorphism (4) in 13 applied to $M=A$ gives rise to an isomorphism

$$
f \mapsto f(1): \operatorname{Endgr}_{A_{\varepsilon}^{e}}(A) \stackrel{\sim}{\rightarrow} \operatorname{Cen}^{\varepsilon}(A)
$$

of graded algebras. Also, we have

$$
\begin{gathered}
\operatorname{Cen}^{\varepsilon}(A)_{\lambda}=\left\{a_{\lambda} \in A_{\lambda} \mid b_{\mu} a_{\lambda}=\varepsilon(\mu, \lambda) a_{\lambda} b_{\mu}, \forall \mu \in \Delta, \forall b_{\mu} \in A_{\mu}\right\}, \\
\operatorname{Cen}^{\varepsilon}(A)=\left\{a \in A \mid z \cdot a=0, \forall z \in \operatorname{ker} \phi_{A}\right\},
\end{gathered}
$$

by $13(1)$ and $13(3)$. Note that $\operatorname{Cen}^{\varepsilon}(A)_{0}$ does not depend on the choice of $\varepsilon$ and is equal to $\operatorname{Cen}(A)_{0}$, the zero-component of the center $\operatorname{Cen}(A)$ of the underlying ungraded ring of $A$. We say that $A$ is $\varepsilon$-central if $\operatorname{Cen}^{\varepsilon}(A)$ is the trivial $R$-algebra. This amounts to saying that both of the following two conditions are satisfied:

$1^{\circ} \operatorname{Cen}^{\varepsilon}(A)=\operatorname{Cen}(A)_{0}$ (which depends on $\varepsilon$ ).

$2^{\circ}$ The canonical map $R \rightarrow \operatorname{Cen}(A)_{0}$ is bijective (which does not depend on $\varepsilon$ ). An example is given by the following proposition:

Proposition. Let $P=\bigoplus_{\lambda \in \Delta} P_{\lambda}$ be a graded $R$-module of type $\Delta$. Assume that $\Delta$ is a group and that the underlying ungraded $R$-module of $P$ is faith- 
ful, finitely generated, and projective. Then the graded $R$-algebra $\operatorname{Endgr}_{R}(P)$ is E-central.

Proof. Since $\Delta$ is a group and $P$ is finitely generated, we have $\operatorname{Endgr}_{R}(P)=$ $\operatorname{End}_{R}(P)$ as ungraded $R$-algebras (cf. $[4, \S 11$, no 6, Remark]). Also the assumption on $P$ implies that $\operatorname{End}_{R}(P)$ is central (in the sense that the structural homomorphism $R \rightarrow \operatorname{End}_{R}(P)$ induces the isomorphism onto the center). Hence we conclude by the following observation, the verification of which is straightforward: For an arbitrary graded $R$-module $M=\bigoplus_{\lambda \in \Delta} M_{\lambda}$ of type $\Delta$, we have $\operatorname{Cen}^{\varepsilon}\left(\operatorname{Endgr}_{R}(M)\right)=\operatorname{Cen}\left(\operatorname{Endgr}_{R}(M)\right)_{0}$, in other words, we have $\operatorname{Cen}^{\varepsilon}$ $\left(\operatorname{Endgr}_{R}(M)\right)_{\lambda}=0$ for $\lambda \neq 0$.

\section{§3. $\varepsilon$-Separable Algebras}

16. Let $R$ be a commutative ring, $\Delta$ an additive monoid whose elements are regular, $\varepsilon$ a commutation factor over $\Delta$ with value in $R$, and $A=\bigoplus_{\lambda \in \Delta} A_{\lambda}$ a graded $R$-algebra of type $\Delta$. Recall (cf. 12) that we have a similar algebra $A_{\varepsilon}^{e}$ operating from left on the underlying graded $R$-module of $A$, and consider the following class of algebras:

Definition. A graded $R$-algebra $A=\bigoplus_{\lambda \in \Delta} A_{\lambda}$ of type $\Delta$ is said to be $\varepsilon$ separable if the ungraded left $A_{\varepsilon}^{e}$-module structure on $A$ is projective.

We begin with giving some criteria for the $\varepsilon$-separability of $A$. Recall from 12 that the multiplication of $A$ induces a map $\phi_{A}: A_{\varepsilon}^{e} \rightarrow A$ which is in fact $A_{\varepsilon}^{e}$-linear. Viewing $\phi_{A}$ as an morphism in the category $A$-Mod- $A$ (cf. 11), we construct a morphism $\left(\phi_{A}\right)_{\varepsilon}^{A}:\left(A_{\varepsilon}^{e}\right)_{\varepsilon}^{A} \rightarrow A_{\varepsilon}^{A}$ in $R$-Mod (cf. 13). Looking at the underlying map of $\left(\phi_{A}\right)_{\varepsilon}^{A}$, we call an element in the fiber of $\left(\phi_{A}\right)_{\varepsilon}^{A}$ over $1 \in A_{\varepsilon}^{A}$ an $\varepsilon$-separability idempotent over $A$. In view of 13 (3), this amounts to saying that:

Definition. An e-separability idempotent over a graded $R$-algebra $A=$ $\bigoplus_{\lambda \in \Delta} A_{\lambda}$ is an element $e$ of $A_{\varepsilon}^{e}$ satisfying the following two conditions:

$$
\begin{gathered}
\phi_{A}(\boldsymbol{e})=1, \\
z \boldsymbol{e}=0 \quad \text { for all } z \in \operatorname{ker} \phi_{A} .
\end{gathered}
$$

Then, as in [2, III-1.6] and [8, (2.10)], we have:

Proposition. The following conditions are equivalent:

(i) $A$ is e-separable.

(ii) The R-linear map $\left(\phi_{A}\right)_{\varepsilon}^{A}:\left(A_{\varepsilon}^{e}\right)_{\varepsilon}^{A} \rightarrow A_{\varepsilon}^{A}$ is surjective. 
(iii) There exists an E-separability idempotent over $A$.

(iv) There exists an $A_{\varepsilon}^{e}$-linear section $A \rightarrow A_{\varepsilon}^{e}$ of $\phi_{A}$.

In particular, the criterion (iii) tells us that

COROLlaRY 1. If $A$ is $\varepsilon$-separable, $\varphi^{*} A$ is $\varphi \varepsilon$-separable for any scalar extension $\varphi: R \rightarrow S$.

Also, using the criterion (ii) and standard arguments as in [5, II, §3], we have the following corollary in which we write $\varepsilon_{p}$ and $A_{p}$ instead of $\varphi \varepsilon$ and $\varphi^{*} A$, respectively, for $\varphi: R \rightarrow R_{p}$ the canonical homomorphism of localization at any prime ideal $\boldsymbol{p}$ of $R$ :

Corollary 2. Let $A=\bigoplus_{\lambda \in \Delta} A_{\lambda}$ be a graded R-algebra of type $\Delta$ whose underlying ungraded $R$-module is finitely presented. Then $A$ is $\varepsilon$-separable if one of the following two conditions are satisfied:

(i) The localization $A_{m}$ is $\varepsilon_{m}$-separable for all maximal ideal $\boldsymbol{m}$ of $\boldsymbol{R}$.

(ii) The scalar extension $\varphi^{*} A$ is $\varphi \varepsilon$-separable for some faithfully flat base change $\varphi: R \rightarrow S$ and the underlying ungraded $R$-module of $A_{\varepsilon}^{A}$ is finitely generated.

Finally, let $e \in A_{\varepsilon}^{e}$ be an $\varepsilon$-separability idempotent. Then we have $\mathbf{e} \cdot A \subset$ $\operatorname{Cen}^{\varepsilon}(A)$, since $z \cdot(\boldsymbol{e} \cdot a)=(z e) \cdot a=0$ for all $z \in \operatorname{ker} \phi_{A}$ and all $a \in A$ (cf. (2), 15 (4)). Moreover we have $\mathbf{e} \cdot a=a$ for $a \in \operatorname{Cen}^{\varepsilon}(A)$, since $e \cdot a-a=(e-1) \cdot a \in$ $\left(\operatorname{Ker} \phi_{A}\right) \cdot a$ (cf. (1), $\left.15(4)\right)$. These formulas can be read that an $\varepsilon$-separability idempotent e defines an $R$-linear retraction $b \mapsto \mathbf{e} \cdot b$ for the inclusion $\operatorname{Cen}^{\varepsilon}(A) \rightarrow$ $A$. Thus we have

Corollary 3. If $A$ is e-separable, $\operatorname{Cen}^{\varepsilon}(A)$ is a direct factor of $A$ as ungraded $R$-modules.

17. An example of the $\varepsilon$-separable algebras is given by the endomorphism algebra of a suitable graded module. Namely:

Proposition. Let $P=\bigoplus_{\lambda \in \Delta} P_{\lambda}$ be a graded $R$-module of type $\Delta$. Assume that $\Delta$ is a group and that the underlying ungraded $R$-module of $P$ is faithful, finitely generated, and projective. Then the graded $R$-algebra $\operatorname{Endgr}_{R}(P)$ is $\varepsilon$ separable. 
ProOF. Since $\Delta$ is a group and $P$ is finitely generated, we have $\operatorname{Endgr}_{R}(P)=$ $\operatorname{End}_{R}(P)$ as ungraded $R$-algebras (cf. $[4, \S 11$, no 6, Remark]). Hence the underlying ungraded $R$-module of $\operatorname{Endgr}_{R}(P)$ is identified with the tensor product $P \otimes_{R} P^{*}$ which is finitely generated and projective by assumption. In particular, the assumption in the corollary 2 in $\mathbf{1 6}$ is satisfied. Note that the $P_{\lambda}$ 's are also finitely generated and projective since they are direct factors in $P$. Thus, after localization, we are reduced to the case where $P$ is non-zero free with base $\left(e_{1}, \ldots, e_{n}\right)$ consisting of homogeneous elements. In this case, denote by $e_{i j}$ the endmorphism of $P$ sending $e_{i}$ to $e_{j}$ and annihilating all the other $e_{k}$ 's. Fix an index $\alpha$ and put

$$
\boldsymbol{e}:=\sum_{1 \leq i \leq n} e_{i \alpha} \otimes e_{\alpha i}^{o \varepsilon} \in \operatorname{Endgr}_{R}(P)_{\varepsilon}^{e} .
$$

Then, we see by simple calculation that $\mathbf{e}$ is an $\varepsilon$-separability idempotent over $\operatorname{Endgr}_{R}(P)$.

18. Let $I$ be an ideal of $R$. Denote by $\varepsilon / I$ the composite $\Delta \times \Delta \stackrel{\varepsilon}{\rightarrow} R \stackrel{\varphi}{\rightarrow} R / I$, where $\varphi$ is the canonical surjection. $\varepsilon / I$ is precisely the commutation factor $\varphi \varepsilon$ defined in 10 (2). If $A=\bigoplus_{\lambda \in \Delta} A_{\lambda}$ is a graded $R$-algebra of type $\Delta$, denote by $I A$ the subgroup of $A$ generated by all the $r a$ 's for $r \in I$ and $a \in A$, which is in fact a graded two-sided ideal of $A$. Thus the quotient of $A$ by $I A$ carries a grading of type $\Delta$, as well as the $(R / I)$-algebra structure. By the notation $A / I A$, we understand the so constructed graded $(R / I)$-algebra. This can be considered also as the algebra $\varphi^{*} A$ derived from $A$ by the scalar extension $\varphi: R \rightarrow R / I$. Denote by $R_{R}(A / I A)$ the graded $R$-algebra of type $\Delta$ derived from $A / I A$ by the scalar restriction $R \rightarrow R / I$. Note that we have a homomorphism $\pi: A \rightarrow{ }_{R}(A / I A)$ of graded $R$-algebras, whose underlying homomorphism of graded rings is the canonical surjection. $\pi$ is precisely the canonical morphism $A \rightarrow \varphi_{*} \varphi^{*} A$ associating to the base change $\varphi: R \rightarrow R / I$. Then, we have the following proposition which is proved by generalizing $[2, \mathrm{III}-1.8]$ in our situation:

Propsoition. Let $A=\bigoplus_{\lambda \in \Delta} A_{\lambda}$ be a graded R-algebra of type $\Delta$, and I be an ideal of $R$. Assume that $A$ is $\varepsilon$-separable. Then the graded $(R / I)$-algebra $A / I A$ is $\varepsilon / I$-separable, and the graded $(\varepsilon / I)$-center $\operatorname{Cen}^{\varepsilon / I}(A / I A)$ has the same underlying graded ring as the image of $\operatorname{Cen}^{\varepsilon}(A)$ under the canonical homomorphism $\pi: A \rightarrow$ ${ }_{R}(A / I A)$.

19. Let $\left(A^{i}\right)_{i}$ be a finite family of graded $R$-algebras $A^{i}=\bigoplus_{\lambda \in \Delta} A_{\lambda}^{i}$ of type $\Delta$. Then the product $A:=\Pi_{i} A^{i}$ acquires a grading with $\lambda$-component $\Pi_{i} A_{\lambda}^{i}$; this 
follows from the fact that a finite product coincides with a direct sum. By construction, the projection $\operatorname{pr}^{i}: a \mapsto a^{i}$ onto any component $A^{i}$ is a homomorphism of graded algebras. As in [2, III-2.20], we have

Proposition. The product $A$ is e-separable if and only if so are all the components $A^{i}$ s.

20. The following proposition is important for the description of the property " $\varepsilon$-Azumaya" (cf. 32), and proved in the same way as in [2, III-2.8]:

Proposition. Let $A=\bigoplus_{\lambda \in \Delta} A_{\lambda}$ and $B=\bigoplus_{\lambda \in \Delta} B_{\lambda}$ be two graded R-algebras of type $\Delta$. Assume that the underlying ungraded $R$-module of $B$ is faithful, finitely generated and projective, and that $A \otimes_{R}^{\varepsilon} B$ is $\varepsilon$-separable. Then $A$ is $\varepsilon$-separable.

21. Let $A=\bigoplus_{\lambda \in \Delta} A_{\lambda}$ and $B=\bigoplus_{\lambda \in \Delta} B_{\lambda}$ be two graded $R$-algebras of type $\Delta$. Observe that the induced homomorphism $\operatorname{Cen}^{\varepsilon}(A) \otimes_{R}^{\varepsilon} \operatorname{Cen}^{\varepsilon}(B) \rightarrow A \otimes_{R}^{\varepsilon} B$ by inclusions is factored by $\operatorname{Cen}^{\varepsilon}\left(A \otimes_{R}^{\varepsilon} B\right)$. Thus we have a homomorphism $\operatorname{Cen}^{\varepsilon}(A) \otimes_{R}^{\varepsilon} \operatorname{Cen}^{\varepsilon}(B) \rightarrow \operatorname{Cen}^{\varepsilon}\left(A \otimes_{R}^{\varepsilon} B\right)$ of graded algebras, which we say canonical. Then, the argument in [2, III-2.6] yields the following generalization:

Proposition. Assume that both $A$ and $B$ are $\varepsilon$-separable. Then so is $A \otimes_{R}^{\varepsilon} B$ and the canonical homomorphism $\operatorname{Cen}^{\varepsilon}(A) \otimes_{R}^{\varepsilon} \operatorname{Cen}^{\varepsilon}(B) \rightarrow \operatorname{Cen}^{\varepsilon}\left(A \otimes_{R}^{\varepsilon} B\right)$ is an isomorphism.

22. From now on until 26, we propose to introduce the notion of "semisimplicity" for graded rings and modules. Let $\Delta$ be an additive monoid whose elements are regular and let $A=\bigoplus_{\lambda \in \Delta} A_{\lambda}$ be a graded ring of type $\Delta$. Recall from 2 the category $A$-Mod of graded left $A$-modules of type $\Delta$ with morphisms $A$-homomorphisms of degree zero. Note that $A$-Mod can be considered as an abelian category in the natural way. Also $A$-Mod admits arbitrary direct sum which is preserved by the forgetful functor with respect to the gradings. Therefore the terms "sum" and "direct sum" have no ambiguities. Moreover it should be noted that so is the term "projective". Indeed, we have the following proposition which is proved along the same line as [8, (1.11)]:

Proposition. Let $M=\bigoplus_{\lambda \in \Delta} M_{\lambda}$ be a graded left A-module of type $\Delta$. Then the following conditions are equivalent:

(i) The underlying ungraded left A-module of $M$ is projective.

(ii) $M$ is a projective object in the abelian category $A$-Mod. 
23. We say that a graded left $A$-module $M=\bigoplus_{\lambda \in \Delta} M_{\lambda}$ is graded simple if $M$ is not zero and has no proper graded $A$-submodule. Then, we have the following theorem which is proved along the same line as the ungraded case (cf. $[4, \S 3$, no 3 , Th. 1]):

THEOREM. Let $M=\bigoplus_{\lambda \in \Delta} M_{\lambda}$ be a graded left A-module of type $\Delta$, and $\left(M_{i}\right)_{i \in I}$ be a family of graded submodules of $M$. Assume that all the $M_{i}$ 's are graded simple and that $M$ is the sum of those. Then, for any graded submodule $N$ of $M$, there exists a subset $J$ of I such that $N$ is the direct sum of the $M_{j}$ 's for $j \in J$.

24. A graded submodule $\mathbf{N}$ of $\mathbf{M}$ is said to be a graded direct factor of $M$ if there exists a graded submodule $N^{\prime}$ of $M$ such that $M$ is the direct sum of $N$ and $N^{\prime}$. Similarly to the ungraded case [cf. [4, VIII, Prop. 7]), the theorem in 23 suggests the following definition:

Definition. Let $M=\bigoplus_{\lambda \in \Delta} M_{\lambda}$ be a graded left A-module of type $\Delta$. We say that $M$ is graded semi-simple if it satisfies the following conditions which are equivalent:

(i) $M$ is the sum of a family of graded simple graded submodules.

(ii) $M$ is the direct sum of a family of graded simple graded submodules.

(iii) Any graded submodule of $M$ is a graded direct factor of $M$.

Note that, in the case where the underlying ungraded $A$-module of $M$ is finitely generated, we may assume the family in the definition to be finite.

25. We say that $A$ is graded semi-simple if every $M \in A$-Mod is projective (cf. 22). A graded semi-simplicity of $A$ implies that every $M \in A$-Mod is graded semi-simple since, for any graded submodule $N$ of $M$, the projectivity of $M / N$ implies that $N$ is a graded direct factor of $M$ (cf. 22, 24). Conversely:

Proposition. Assume that $\Delta$ is a group. Then the following conditions are equivalent:

(i) $A$ is graded semi-simple.

(ii) Every $M \in A$-Mod is graded semi-simple.

(iii) The statement in (ii) holds for $M=A_{s}$, the graded left A-module derived from $A$. 
Proof. It remains to show that (iii) implies (i). Since $\Delta$ is a group, there exists a graded free left $A$-module $F \in A$-Mod and a epimorphism $\pi: F \rightarrow M$ in $A$-Mod (cf. [4, II, $\S 11$, no 2, Example (3)]). (iii) implies that $F$ is graded semisimple. Thus $\pi$ splits, and hence $M$ is projective.

26. We say that $A$ is graded quasi-simple if it is not zero and has no proper graded two-sided ideal. Observe that, if this is the case, the zero component $\operatorname{Cen}(A)_{0}$ of the center $\operatorname{Cen}(A)$ (cf. [4, II, $\S 11$, no 3, Prop. 5, Cor.]) is a field. $A$ is said to be graded simple if it is graded semi-simple (cf. 24) and graded quasisimple. By a maximal graded left ideal of $A$, we understand a maximal element of the set of proper graded left ideals of $A$ ordered by inclusions. Denote by $\mathscr{R}(A)$ the intersection of maximal graded left ideals of $A$. Similarly to the ungraded case (cf. [4, VIII, §6, no 3]), we see that

a) $x \in \mathscr{R}(A)$ if and only if $x \in \operatorname{ann}_{A}(S)$ for any graded simple $S \in A$-Mod.

b) $\mathscr{R}(A)$ is the minimal element of the set $\left\{\operatorname{ann}_{A}(M) \mid M \in A\right.$-Mod, $M$ is graded semi-simple $\}$ (any element of which is a graded two-sided ideal of $A$ ) ordered by inclusions.

In particular, $\mathscr{R}(A)$ is a graded two-sided ideal of $A$. We say that $A$ is graded left Artinian if any non-empty set consisting of graded left ideal of $A$ has a minimal element with respect to the inclusions. For example, this is the case if the underlying ungraded ring of $A$ is left Artinian. By the canonical argument (cf. [4, VIII, no 4, Th. 4]), we see that if $A$ is graded left Artinian there exists a finite family $\left(\boldsymbol{m}_{i}\right)_{1 \leq i \leq n}$ of maximal graded left ideals of $A$ such that $\mathscr{R}(A)=\bigcap_{1 \leq i \leq n} \boldsymbol{m}_{i}$.

Proposition. Assume that $\Delta$ is a group and that $A$ is graded quasi-simple and graded left Artinian. Then $A$ is graded simple.

Proof. It suffices to show that $A$ is graded semi-simple (cf. 25). Indeed we have $\{0\}=\mathscr{R}(A)=\bigcap_{1 \leq i \leq n} \boldsymbol{m}_{i}$ for some maximal graded left ideals $\boldsymbol{m}_{1}, \ldots, \boldsymbol{m}_{n}$ of $A$. It follows that the canonical map $\varphi: A \rightarrow \bigoplus_{1 \leq i \leq n} A / \boldsymbol{m}_{i}$ is injective. $\varphi$ is a morphism in $A$-Mod whose target is graded semi-simple. Hence so is $A$.

27. Our next aim is $\mathbf{2 9}$ which asserts the graded simplicity for some $\varepsilon$ separable algebras over a field. We begin with the following proposition which is proved in the same way as in $[8,(5.7)$ (ii)]:

Proposition. Let $R$ be a field, $\Delta$ an additive monoid whose elements are regular, $\varepsilon$ a commutation factor over $\Delta$ with value in $R$, and $A=\bigoplus_{\lambda \in \Delta} A_{\lambda} a$ 
graded R-algebra of type $\Delta$. Assume that $A$ is E-separable. Then the underlying graded ring of $A$ is graded semi-simple.

28. Proposition. Let $(R, \Delta, \varepsilon)$ be as in 27 and $A=\bigoplus_{\lambda \in \Delta} A_{\lambda}$ be a graded $R$-algebra of type $\Delta$. Assume that $\Delta$ is a group, and that $A$ is finite-dimensional and $\varepsilon$-separable. Then $A$ is isomorphic to a finite product $\prod_{i} A^{i}$, where each $A^{i}$ is a graded $R$-algebra of type $\Delta$ which is finite-dimensional, $\varepsilon$-separable, and graded simple.

Proof. The adjective finite-dimensional (resp. graded simple) refers to the underlying ungraded $R$-vector space (resp. graded ring) of the algebra in question. The graded $R$-algebra $A_{\varepsilon}^{e}$ is also finite-dimensional and $\varepsilon$-separable by 21 . Hence $A_{\varepsilon}^{e}$ is graded semi-simple by 27. It follows that the left $A_{\varepsilon}^{e}$-module $A$ decomposes into a direct sum $A^{1} \oplus \cdots \oplus A^{r}$ of graded simple submodules (cf. 24, 25). By the definition of $A_{\varepsilon}^{e}$-module structure on $A$ (cf. 12), each $A^{i}$ is a two-sided ideal of $A$. This implies that $A^{i}$ inherits from $A$ a structure of graded $R$-algebra of type $\Delta$ and that the map $\varphi: A^{1} \times \cdots A^{r} \rightarrow A$ induced by the addition gives rise to an isomorphism. Clearly $A^{i}$ is finite-dimensional. The $\varepsilon$-separability of $A^{i}$ follows from 19. We claim that $A^{i}$ is graded simple. Since $A^{i}$ is finite-dimensional, it suffices to show the graded quasi-simplicity (cf. 26). Indeed, a graded two-sided ideal $\boldsymbol{m}$ of $A^{i}$ is also that of $A$. It follows that $\boldsymbol{m}$ is the sum $\sum_{j \in J} A^{j}$ for certain $J \subset[1, r]$ (cf. 23). If $i \in J$ then $\boldsymbol{m}=A^{i}$. Otherwise we have $\boldsymbol{m}=\{0\}$.

29. Corollary. Let $(R, \Delta, \varepsilon)$ be as in 27 and $A=\bigoplus_{\lambda \in \Delta} A_{\lambda}$ be a graded $R$-algebra of type $\Delta$. Assume that $\Delta$ is a group, and that $A$ is finite-dimensional, $\varepsilon$-separable, and $\varepsilon$-central. Then the underlying graded ring of $A$ is graded simple.

Proof. $A$ is isomorphic to the product $\prod_{i \in I} A^{i}$ of a finite family of graded simple graded algebras $A^{i}$ (cf. 28). The fact that $\operatorname{Cen}^{\varepsilon}\left(\prod_{i \in I} A^{i}\right)=\prod_{i \in I} \operatorname{Cen}^{\varepsilon}\left(A^{i}\right)$ is clear from the formula 15 (3). This is isomorphic to $R$ which is a field by assumption. It follows that the set $I$ is a singleton, which shows our assertion.

\section{§4. $\varepsilon$-Azumaya Algebras}

30. Let $R$ be a commutative ring, $\Delta$ an additive monoid whose elements are regular, and $\varepsilon$ a commutation factor over $\Delta$ with value in $R$. For any graded $R$-algebra $A=\bigoplus_{\lambda \in \Delta} A_{\lambda}$ of type $\Delta$, we have an adjoint situation (cf. 1 )

$$
\left(\varphi^{\varepsilon} ; ?_{\varepsilon}^{A}, A \otimes_{R}^{\varepsilon} ? ; A \text {-Mod- } A, R \text {-Mod }\right)
$$


which is identified with the situation $\left(\varphi^{\mathrm{gr}} ; \operatorname{Homgr}_{A_{\varepsilon}^{e}}(A, \cdot), A \otimes_{R}^{\varepsilon} ; A_{\varepsilon}^{e}\right.$-Mod, $R$-Mod $)$ associated to the graded $\left(A_{\varepsilon}^{e} ; R\right)$-bimodule structure on $A$ (cf. 2 (1), 11 (2) and 13 (4)). For the convenience, we shall describe the situation (1) directly. $A$-Mod- $A$ is the category of graded $(A ; A)$-bimodules of type $\Delta$, in the sense of 11, and $R$-Mod is that of graded $R$-modules. The functor

$$
?_{\varepsilon}^{A}: A \text {-Mod- } A \rightarrow R \text {-Mod }
$$

is the one defined in 13 by the formula $\left(M_{\varepsilon}^{A}\right)_{\lambda}=\left\{x_{\lambda} \in M_{\lambda} \mid a_{\mu} x_{\lambda}=\varepsilon(\mu, \lambda) x_{\lambda} a_{\mu}\right.$, $\left.\forall \mu \in \Delta, \forall a_{\mu} \in A_{\mu}\right\}$ for $M \in A$-Mod- $A$ and $\lambda \in \Delta$. The functor

$$
A \otimes_{R}^{\varepsilon} ?: R \text {-Mod } \rightarrow A \text {-Mod- } A
$$

assigns to $E \in R$-Mod the graded $(A ; A)$-bimodule $A \otimes_{R}^{\varepsilon} E$ whose underlying graded $R$-module is the ordinary tensor product $A \otimes_{R} E$ with the total grading, and the $(A ; A)$-bimodule structure is defined by the formulas $a \cdot(b \otimes e):=$ $(a b) \otimes e$ and $\left(b \otimes e_{v}\right) \cdot a_{\lambda}:=\varepsilon(v, \lambda)\left(b a_{\lambda}\right) \otimes e_{v}$ for $a, b \in A, e \in E, \lambda, v \in \Delta, a_{\lambda} \in A_{\lambda}$, and $e_{v} \in E_{v}$ (cf. 11 (4)). If $M \in A$-Mod- $A$, the formula $\eta_{M}^{\varepsilon}(a \otimes x):=a x$ defines a morphism

$$
\eta_{M}^{\varepsilon}: A \otimes_{R}^{\varepsilon}\left(M_{\varepsilon}^{A}\right) \rightarrow M
$$

in $A$-Mod- $A$. Also if $E \in R$-Mod and if $e \in E$, the element $1 \otimes e$ of $A \otimes_{R} E$ in fact belongs to $\left(A \otimes_{R}^{\varepsilon} E\right)_{\varepsilon}^{A}$. Denoting this by $\xi_{E}^{\varepsilon}(e)$, we obtain a morphism

$$
\xi_{E}^{\varepsilon}: E \rightarrow\left(A \otimes_{R}^{\varepsilon} E\right)_{\varepsilon}^{A}
$$

in $R$-Mod. Then we have an isomorphism

$$
\varphi^{\varepsilon}(M, E): A-\operatorname{Mod}-A\left(A \otimes_{R}^{\varepsilon} E, M\right) \stackrel{\sim}{\rightarrow} R-\operatorname{Mod}\left(E, M_{\varepsilon}^{A}\right)
$$

by sending $f \in A$-Mod- $A\left(A \otimes_{R}^{\varepsilon} E, M\right)$ to $\left(f_{\varepsilon}^{A}\right) \circ \xi_{E}^{\varepsilon} \in R-\operatorname{Mod}\left(E, M_{\varepsilon}^{A}\right)$. The inverse is given by sending $g \in R-\operatorname{Mod}\left(E, M_{\varepsilon}^{A}\right)$ to $\eta_{M}^{\varepsilon} \circ\left(A \otimes_{R}^{\varepsilon} g\right) \in A-\operatorname{Mod}-A\left(A \otimes_{R}^{\varepsilon} E, M\right)$. In our situation, it should be noted that both $A$-Mod- $A$ and $R$-Mod admit products given by $\otimes_{A}$ and $\otimes_{R}$, respectively (cf. 8). The functor $A \otimes_{R}^{\varepsilon}$ preserves these products since, for any $E$ and $F$ in $R$-Mod, an isomorphism $\Phi(E, F)$ : $\left(A \otimes_{R}^{\varepsilon} E\right) \otimes_{A}\left(A \otimes_{R}^{\varepsilon} F\right) \stackrel{\sim}{\rightarrow} A \otimes_{R}^{\varepsilon}\left(E \otimes_{R} F\right)$ in $A$-Mod- $A$ is given by the formula $\Phi(E, F)\left(\left(a \otimes e_{\lambda}\right) \otimes\left(b_{\mu} \otimes f\right)\right):=\varepsilon(\lambda, \mu)\left(a b_{\mu}\right) \otimes\left(e_{\lambda} \otimes f\right) \quad$ for $\quad a \in A, \quad f \in F, \quad \lambda$, $\mu \in \Delta, e_{\lambda} \in E_{\lambda}$ and $b_{\mu} \in A_{\mu}$.

31. As in 3, the following definition is valid: 
Definition. Let $A=\bigoplus_{\lambda \in \Delta} A_{\lambda}$ be a graded $R$-algebra of type $\Delta$. We say that $A$ is $\varepsilon$-Azumaya if it satisfies the following conditions which are equivalent.

(i) The functor $?_{\varepsilon}^{A}: A$-Mod- $A \rightarrow R$-Mod is an equivalence:

(ii) The functor $A \otimes_{R}^{\varepsilon}$ ?:R-Mod $\rightarrow A$-Mod- $A$ is an equivalence.

(iii) All the morphisms $\eta_{M}^{\varepsilon}: A \otimes_{R}^{\varepsilon}\left(M_{\varepsilon}^{A}\right) \rightarrow M$ and $\xi_{E}^{\varepsilon}: E \rightarrow\left(A \otimes_{R}^{\varepsilon} E\right)_{\varepsilon}^{A}$, where $M \in A$-Mod- $A$ and $E \in R$-Mod, are isomorphisms.

Note that, if this is the case, the functor $?_{\varepsilon}^{A}: A$-Mod- $A \rightarrow R$-Mod also preserves products given by $\otimes_{A}$ and $\otimes_{R}$.

32. We will establish some criteria for an $\varepsilon$-Azumaya in 33. We begin with the following proposition which is proved in the same way as in $[8,(6.2)]$ using 18 and 29:

Proposition. Assume that $\Delta$ is a group, and that $A$ is $\varepsilon$-central, E-separable, and finitely generated as an ungraded $R$-module. Then the following two sets coincide:

a) The maximal elements of the set $\{\mathscr{M} \mid$ graded two-sided ideal of $A, \mathscr{M} \neq$ A\}.

b) The $\boldsymbol{m} A$ 's, where $\boldsymbol{m}$ runs in the set of maximal ideals of $R$.

33. TheOrem. Let $A=\bigoplus_{\lambda \in \Delta} A_{\lambda}$ be a graded R-algebra of type $\Delta$. Assume that $\Delta$ is a group. Then the following conditions are equivalent:

(i) $A$ is e-Azumaya.

(ii) The underlying ungraded $R$-module of $A$ is finitely generated, projective, and faithful, and the canonical representation $A_{\varepsilon}^{e} \rightarrow \operatorname{Endgr}_{R}(A)$ is an isomorphism.

(iii) $A$ is E-central and is a generator as a ungraded left $A_{\varepsilon}^{e}$-module.

(iv) $A$ is e-central, E-separable, and the underlying ungraded $R$-module of $A$ is finitely generated.

(v) There exists an isomorphism $A \otimes_{R}^{\varepsilon} B \stackrel{\sim}{\rightarrow} \operatorname{Endgr}_{R}(P)$ of graded $R$-algebras for some graded $R$-algebra $B=\bigoplus_{\lambda \in \Delta} B_{\lambda}$ and a graded $R$-module $P=\bigoplus_{\lambda \in \Delta} P_{\lambda}$ whose underlying ungraded $R$-module is finitely generated, projective, and faithful.

Proof. (i) $\Leftrightarrow$ (ii) $\Leftrightarrow$ (iii): This follows from the theorem in 5 and criteria (vi) and (vii) in 4 .

(ii) $\Leftrightarrow(\mathrm{v}): B:=A^{o \varepsilon}$ and $P:=A$ are what we want.

(v) $\Rightarrow$ (iv): Since $\Delta$ is a group, the isomorphism $A \otimes_{R}^{\varepsilon} B \stackrel{\sim}{\rightarrow} \operatorname{Endgr}_{R}(P)$ induces an isomorphism $A \otimes_{R} B \stackrel{\sim}{\rightarrow} \operatorname{End}_{R}(P)$ of underlying ungraded $R$-modules (cf. [4, II, $\S 11$, no 6 , Remark]). Thus the underlying ungraded $R$-modules of $A$ 
and $B$ are finitely generated, projective, and faithful (cf. [2, I-6.1]). By 20, we see that $A$ and $B$ are $\varepsilon$-separable. Then, by 21 and 15, the underlying ungraded $R$-modules of $\operatorname{Cen}^{\varepsilon}(A)$ and $\operatorname{Cen}^{\varepsilon}(B)$ become invertible. However, $R$ is a direct factor in $A$ (resp. $B$ ) (cf. [2, III-2.17]), and contained in $\operatorname{Cen}^{\varepsilon}(A)\left(\right.$ resp. $\operatorname{Cen}^{\varepsilon}(B)$ ). It follows that $\operatorname{Cen}^{\varepsilon}(A) \simeq R \simeq \operatorname{Cen}^{\varepsilon}(B)$.

(iv) $\Rightarrow$ (iii): This follows from the same argument as in the proof of $(1) \Rightarrow(2)$ in [2, III-4.1], by using 32 .

\section{§5. Automorphisms}

34. Let $R$ be a commutative ring and $\Delta$ be an additive monoid. If $M=$ $\bigoplus_{\lambda \in \Delta} M_{\lambda}$ is a graded $R$-module of type $\Delta$ and if $\boldsymbol{p} \in \operatorname{Spec} R$, the localization $M_{\boldsymbol{p}}$ is a graded $R_{p}$-module of type $\Delta$. The canonical map $M \rightarrow M_{p}, u \mapsto u_{p}$, is compatible with taking homogeneous components in the sense that we have $\left(u_{\boldsymbol{p}}\right)_{\lambda}=\left(u_{\lambda}\right)_{\boldsymbol{p}}$ for $u \in M, \boldsymbol{p} \in \operatorname{Spec} R$, and $\lambda \in \Delta$. Hence, for such $(u, \boldsymbol{p}, \lambda)$, we have

$$
u_{p} \text { is homogeneous of degree } \lambda \Leftrightarrow\left(u-u_{\lambda}\right)_{p}=0 .
$$

Note that, if $(u, \lambda)$ is fixed, such $p$ 's form an open subset in $\operatorname{Spec} R$. Also we see from (1) that an element $u \in M$ is homogeneous of degree $\lambda$ if so are $u_{p}$ for all $p \in \operatorname{Spec} R$. We say that an element $u \in M$ is locally homogeneous if $u_{p}$ is homogeneous for all $p \in \operatorname{Spec} R$.

Lemma. Let $u$ be an element of $M$. Assume that $u$ is free and that the submodule $R \cdot u$ of $M$ is graded. Then $u$ is locally homogeneous.

ProOF. Since every homogeneous component $u_{\lambda}$ of $u$ belongs to $R \cdot u$, we have a family $\left(f_{\lambda}\right)_{\lambda \in \Delta}$ of elements of $R$ such that $f_{\lambda} u=u_{\lambda}$. The support of this family is finite and $\sum_{\lambda} f_{\lambda}=1$, since so is $\left(u_{\lambda}\right)_{\lambda \in \Delta}$ and $u$ is free. It follows that any $\boldsymbol{p} \in \operatorname{Spec} R$ admits $\lambda \in \Delta$ such that $f_{\lambda} \notin \boldsymbol{p}$. In this case $u_{\boldsymbol{p}}$ is homogeneous of degree $\lambda$.

35. Let $A=\bigoplus_{\lambda \in \Delta} A_{\lambda}$ be a graded $R$-algebra of type $\Delta$, and $\varepsilon$ be a commutation factor over $\Delta$ with value in $R$. Denote by $\operatorname{Aut}(A)$ the automorphism group of $A$ with respect to the graded algebra structure. Note that the homogeneous units form a subgroup of $A^{*}$, the whole of units in $A$. Denote by $h U(A)$ this subgroup. Also the locally homogeneous units form a subgroup of $A^{*}$, which we denote by $\tilde{h} U(A)$. The underlying set of the group $h U(A)$ is identified with the union of $A^{*} \cap A_{\lambda}$ 's for all $\lambda \in \Delta$, which is disjoint provided $A$ is not zero. 
For $\lambda \in \Delta$ and $u_{\lambda} \in A^{*} \cap A_{\lambda}$, the formula

$$
i_{A}^{\varepsilon}\left(u_{\lambda}\right)\left(x_{v}\right):=\varepsilon(v, \lambda) u_{\lambda} x_{v} u_{\lambda}^{-1},
$$

where $v \in \Delta$ and $x_{v} \in A_{v}$, defines an automorphism $i_{A}^{\varepsilon}\left(u_{\lambda}\right)$ of $A$. We call $i_{A}^{\varepsilon}\left(u_{\lambda}\right)$ the $\varepsilon$-inner automorphism of $A$ induced by $u_{\lambda}$. We may regard $i_{A}^{\varepsilon}$ as a map from $h U(A)$ to Aut $(A)$, which is in fact a group homomorphism. By (1) and 15, the kernel of $i_{A}^{\varepsilon}$ is the union $A^{*} \cap \operatorname{Cen}^{\varepsilon}(A)_{\lambda}$ 's for all $\lambda \in \Delta$, while $A^{*} \cap \operatorname{Cen}^{\varepsilon}(A)_{\lambda}$ is in fact $\operatorname{Cen}^{\varepsilon}(A)^{*} \cap \operatorname{Cen}^{\varepsilon}(A)_{\lambda}$. In this way, we obtain an exact sequence

$$
1 \longrightarrow h U\left(\operatorname{Cen}^{\varepsilon}(A)\right) \longrightarrow h U(A) \stackrel{i_{A}^{\varepsilon}}{\longrightarrow} \operatorname{Aut}(A)
$$

of groups.

We now consider the case where $A_{p} \neq 0$ for all $p \in \operatorname{Spec} R$. (This is the case if, for example, $A$ is $\varepsilon$-Azumaya.) Then, for $u \in \tilde{h} U(A)$ and $p \in \operatorname{Spec} R$, the degree of $u_{p}$ is uniquely determined since $u_{p}$ is a non-zero homogeneous element in $A_{p}$. Denoting by $d_{u}(\boldsymbol{p}) \in \Delta$ the degree of $u_{\boldsymbol{p}}$, we have $d_{1}(\boldsymbol{p})=0$ and $d_{u v}(\boldsymbol{p})=$ $d_{u}(\boldsymbol{p})+d_{v}(\boldsymbol{p})$. Moreover, for fixed $u \in \tilde{h} U(A)$ and $\lambda \in \Delta$, the $\boldsymbol{p}$ 's such that $d_{u}(\boldsymbol{p})=\lambda$ form an open subset in $\operatorname{Spec} R$ (cf. 34). Hence, the function $d_{u}$ : Spec $R \rightarrow \Delta$ is locally constant and the map $u \mapsto d_{u}$ is a homomorphism $d: \tilde{h} U(A) \rightarrow \operatorname{Top}(\operatorname{Spec} R, \Delta)$ of monoids. Note that $A^{*} \cap A_{\lambda}$ is precisely the fiber of $d$ over the constant $\lambda$-valued function. It follows that the sequence

$$
1 \rightarrow A_{0}^{*} \rightarrow \tilde{h} U(A) \stackrel{d}{\rightarrow} \operatorname{Top}(\operatorname{Spec} R, \Delta)
$$

is exact. We propose to extend the above homomorphism $i_{A}^{\varepsilon}: h U(A) \rightarrow \operatorname{Aut}(A)$ to $\tilde{h} U(A)$. For $u \in \tilde{h} U(A), v \in \Delta$, and $x_{v} \in A_{v}$, set

$$
i_{A}^{\varepsilon}(u)\left(x_{v}\right):=\sum_{\mu \in \Delta} \varepsilon(v, \mu) u_{\mu} x_{v} u^{-1}
$$

and consider $i_{A}^{\varepsilon}(u)$ as an endomorphism of the underlying graded $R$-module of $A$. We claim that $i_{A}^{\varepsilon}(u)$ is in fact an element of $\operatorname{Aut}(A)$ and that the map $i_{A}^{\varepsilon}: u \mapsto$ $i_{A}^{\varepsilon}(u)$ is a homomorphism. Indeed, since we have $i_{A}^{\varepsilon}(1)=\operatorname{Id}_{A}$ clearly from the definition, it remains to show that $i_{A}^{\varepsilon}$ respects the products and that $i_{A}^{\varepsilon}$ is a homomorphism. Now the question being local, we are reduced to the case where $u$ is homogeneous. Then the assertion is clear since, after localizing at $p \in \operatorname{Spec} R$, we have

$$
i_{A}^{\varepsilon}(u)_{p}\left(z_{v}\right)=\varepsilon\left(v, d_{u}(\boldsymbol{p})\right) u_{\boldsymbol{p}} z_{v} u_{\boldsymbol{p}}^{-1}
$$

for $z_{v} \in\left(A_{p}\right)_{v}$. We see from (4) and (1) that so constructed homomorphism $i_{A}^{\varepsilon}$ : 
$\tilde{h} U(A) \rightarrow \operatorname{Aut}(A)$ is indeed a desired extension. For $u \in \tilde{h} U(A)$, we call $i_{A}^{\varepsilon}(u)$ the $\varepsilon$-inner autmorphism induced by $u$. The condition $i_{A}^{\varepsilon}=\operatorname{Id}_{A}$ on $u \in \tilde{h} U(A)$ is equivalent to $u \in \operatorname{Cen}^{\varepsilon}(A)$, by (4) and 15 (3). By localization, we see that $\operatorname{Cen}^{\varepsilon}(A) \cap \tilde{h} U(A)=\tilde{h} U\left(\operatorname{Cen}^{\varepsilon}(A)\right)$. In this way, we obtain an exact sequence

$$
1 \longrightarrow \tilde{h} U\left(\operatorname{Cen}^{\varepsilon}(A)\right) \longrightarrow \tilde{h} U(A) \stackrel{i_{A}^{\varepsilon}}{\longrightarrow} \operatorname{Aut}(A)
$$

of groups.

36. From now on, we assume that $\Delta$ is a group. We denote by $\operatorname{Picgr}^{\Delta}(R)$ the graded Picard group (cf. 8) of the graded ring of type $\Delta$ derived from $R$ by the trivial grading. In this case, $R$-Mod- $R$ is identified with $R$-Mod in an obvious way. Then, the graded invertible $R$-modules is precisely the graded $R$-modules of type $\Delta$ whose underlying ungraded $R$-modules are invertible, i.e. projective of rank one (cf. 5, [2, II-3.2], [5, II $\S 5$, no 4, Th. 3]). Also $\operatorname{Picgr}^{\Delta}(R)$ is the group of such graded $R$-modules modulo graded $R$-isomorphisms of degree zero. Denote by $\operatorname{Pic}(R)$ the ordinary Picard group of $R$. Then we have two homomorphisms

$$
\operatorname{Picgr}^{\Delta}(R) \rightarrow \operatorname{Pic}(R) \quad \text { and } \quad \operatorname{Pic}(R) \rightarrow \operatorname{Picgr}^{\Delta}(R)
$$

induced by forgetting gradings and adding trivial gradings, respectively. By constructions, the composite $\operatorname{Pic}(R) \rightarrow \operatorname{Picgr}^{\Delta}(R) \rightarrow \operatorname{Pic}(R)$ is the identity. On the other hand, we have two homomorphisms

$$
\sigma: \operatorname{Top}(\operatorname{Spec} R, \Delta) \rightarrow \operatorname{Picgr}^{\Delta}(R) \quad \text { and } \quad \pi: \operatorname{Picgr}^{\Delta}(R) \rightarrow \operatorname{Top}(\operatorname{Spec} R, \Delta),
$$

which are defined as follows: $\sigma$ sends $f \in \operatorname{Top}(\operatorname{Spec} R, \Delta)$ to the class of $R(f) \in$ $R$-Mod, where $R(f)$ is the $R$ with the grading $\left(R(f)_{\lambda}\right)_{\lambda \in \Delta}$ defined by $f$ (cf. [5, II, $\S 4$, no 3, Prop. 15]). Namely, we have $R(f)_{\lambda}=R f_{\lambda}$, where $\left(f_{\lambda}\right)_{\lambda \in \Delta}$ is the orthogonal decomposition of the unit of $R$ (by this we understand that $f_{\lambda}=0$ for almost all $\lambda$, that $\sum_{\lambda} f_{\lambda}=1$, and that $f_{\lambda} f_{\mu}=0$ for $\lambda \neq \mu$ ), characterized by the property

$$
f^{-1}(\lambda)=\left\{\boldsymbol{p} \in \operatorname{Spec} R \mid f_{\lambda} \notin \boldsymbol{p}\right\} \quad \text { for all } \lambda \in \Delta .
$$

From this description of $R(f)$, we see that the map $\sigma: f \mapsto \sigma(f)$ is in fact a homomorphism, since the group $\operatorname{Top}(\operatorname{Spec} R, \Delta)$ can be imbedded into the multiplicative group of the group algebra $R[\Delta]$, by the map sending $f$ to $\sum_{\lambda} f_{\lambda} e^{\lambda}$, where $\left(e^{\lambda}\right)_{\lambda \in \Delta}$ stands for the canonical base of $R[\Delta]$. On the other hand, let $L=\bigoplus_{\lambda \in \Delta} L_{\lambda}$ be a graded invertible $R$-module of type $\Delta$. Then each $L_{\lambda}$ is finitely generated projective, and almost all the $L_{\lambda}$ 's are zero. Thus, for each $\lambda \in \Delta$, we 
have a locally constant function $\boldsymbol{p} \mapsto \operatorname{rk}_{p}\left(L_{\lambda}\right): \operatorname{Spec} R \rightarrow \boldsymbol{Z}$, the rank of $L_{\lambda}$ (cf. [5, II, §5, no 3]). For each $p \in \operatorname{Spec} R$, we have $\sum_{\lambda} \operatorname{rk}_{p}\left(L_{\lambda}\right)=1$, since $\oplus_{\lambda} L_{\lambda}=L$ is of rank one. It follows that there exists unique index $\lambda$ such that $\operatorname{rk}_{p}\left(L_{\lambda}\right)=1$. Denoting by $\lambda_{L}(\boldsymbol{p})$ this index, we obtain a function

$$
\lambda_{L}: \operatorname{Spec} R \rightarrow \Delta,
$$

which is locally constant since so is each $\operatorname{rk}\left(L_{\lambda}\right)$. It can be said that $\lambda_{L}(p)$ is the unique index $\lambda$ such that $\left(L_{\lambda}\right)_{p}$ is not zero, i.e. $\boldsymbol{p} \in \operatorname{Supp}_{R}\left(L_{\lambda}\right)$. From this, we see $\lambda_{L \otimes_{R} M}(\boldsymbol{p})=\lambda_{L}(\boldsymbol{p})+\lambda_{M}(\boldsymbol{p})$, since $L_{\lambda} \otimes_{R} M_{\mu}$ is a direct factor of $\left(L \otimes_{R} M\right)_{\lambda+\mu}$. Clearly $\lambda_{L}$ depends only on the isomorphism class of $L$. Thus $\operatorname{cl}(L) \mapsto \lambda_{L}$ is a homomorphism from $\operatorname{Picgr}^{\Delta}(R)$ to $\operatorname{Top}(\operatorname{Spec} R, \Delta)$. This homomorphism is $\pi$, by definition.

These being said, observe that both of two sequences

$$
0 \rightarrow \operatorname{Top}(\operatorname{Spec} R, \Delta) \stackrel{\sigma}{\rightarrow} \operatorname{Picgr}^{\Delta}(R) \rightleftarrows \operatorname{Pic}(R) \rightarrow 0
$$

and

$$
0 \longrightarrow \operatorname{Pic}(R) \longrightarrow \operatorname{Picgr}^{\Delta}(R) \stackrel{\pi}{\rightleftarrows} \operatorname{Top}(\operatorname{Spec} R, \Delta) \longrightarrow 0
$$

are exact and split.

37. Let $R$ be a commutative ring, $\Delta$ be an additive group, and $\varepsilon$ be a commutation factor over $\Delta$ with value in $R$. If $A=\bigoplus_{\lambda \in \Delta} A_{\lambda}$ is a graded $R$ algebra of type $\Delta$, denote by $\operatorname{Aut}(A)$ the automorphism group of $A$ with respect to the graded algebra structure. For $\alpha \in \operatorname{Aut}(A)$, we define a graded $R$-module $L^{\varepsilon}(\alpha)=\bigoplus_{\lambda \in \Delta} L^{\varepsilon}(\alpha)_{\lambda}$ to be the graded submodule of the underlying graded $R$ module of $A$ with $\lambda$-component

$$
L^{\varepsilon}(\alpha)_{\lambda}:=\left\{x_{\lambda} \in A_{\lambda} \mid \alpha\left(a_{\mu}\right) x_{\lambda}=\varepsilon(\mu, \lambda) x_{\lambda} a_{\mu}, u \in \Delta, a_{\mu} \in A_{\mu}\right\} .
$$

Observe that, for $\alpha, \beta \in \operatorname{Aut}(A)$, the multiplication in $A$ induces a graded $R$ homomorphism

$$
\Phi_{\alpha, \beta}^{\varepsilon}: L^{\varepsilon}(\alpha) \otimes_{R} L^{\varepsilon}(\beta) \rightarrow L^{\varepsilon}(\alpha \beta)
$$

of degree zero. In the notation of 9 , the definition (1) can be read as $L^{\varepsilon}(\alpha)=$ $\left({ }_{\alpha} A_{1}\right)_{\varepsilon}^{A}$. Recall that ${ }_{\alpha} A_{1} \in A$-Mod- $A$ is graded invertible (cf. 9), and that the functor $M \mapsto M_{\varepsilon}^{A}$ preserves products provided $A$ is $\varepsilon$-Azumaya (cf. 31). Hence

THEOREM. If $A$ is E-Azumaya, the underlying ungraded $R$-module of all the $L^{\varepsilon}(\alpha)$ 's are projective of rank one and all $\Phi_{\alpha, \beta}^{\varepsilon}$ 's are isomorphisms. 
38. In general, if $L$ is an $R$-module which is projective of rank one, we denote by $L^{*}$ the generators of $L$. Namely, $L^{*}$ is the set of $u \in L$ 's such that $R u=L$. Therefore, $L^{*}$ is not empty if and only if $L$ is trivial. Also, the map $r \mapsto r u$ is an isomorphism $R \stackrel{\sim}{\rightarrow} L$ for all $u \in L^{*}$. Then, we have the following theorem which is proved in the same way as in $[3,1.3 \mathrm{Th}$. (c)]:

THEOREM. If $A$ is $\varepsilon$-Azumaya, we have

$$
L^{\varepsilon}(\alpha)^{*}=\left\{u \in \tilde{h} U(A) \mid i_{A}^{\varepsilon}(u)=\alpha\right\}
$$

for all $\alpha \in \operatorname{Aut}(A)$.

39. Let $A=\bigoplus_{\lambda \in \Delta} A_{\lambda}$ be a graded $R$-algebra of type $\Delta$. Assume that $A$ is $\varepsilon$-Azumaya. Denote by $\operatorname{Picgr}_{R}(A)$ the subgroup of $\operatorname{Picgr}(A)$ (cf. 9) consisting of the classes $\operatorname{cl}(P)$ represented by objects $P$ in $A$-Mod- $A$ in the sense of 8 . Then we have an isomorphism

$$
\operatorname{cl}(P) \mapsto \operatorname{cl}\left(P_{\varepsilon}^{A}\right): \operatorname{Picgr}_{R}(A) \stackrel{\sim}{\rightarrow} \operatorname{Picgr}^{\Delta}(R)
$$

of groups, since the functor $?_{\varepsilon}^{A}: A$-Mod- $A \rightarrow R$-Mod is an equivalence which preserves the products (cf. 31). By 37, each $\alpha \in \operatorname{Aut}(A)$ defines an element $\psi_{A}^{\varepsilon}(\alpha)$ of $\operatorname{Picgr}^{\Delta}(R)$ by the formula

$$
\psi_{A}^{\varepsilon}(\alpha):=\operatorname{cl}\left(L^{\varepsilon}(\alpha)\right) \in \operatorname{Picgr}^{\Delta}(R) .
$$

Since the map $\alpha \mapsto \psi_{A}^{\varepsilon}(\alpha)$ is the successive composite of the homomorphism $\operatorname{Aut}(A) \rightarrow \operatorname{Picgr}_{R}(A)$ induced by $\varphi_{A}$ in 9 , the isomorphism (1), and the symmetry $z \mapsto-z$ in $\operatorname{Picgr}_{R}(A)$, we thus obtain a homomorphism $\psi_{A}^{\varepsilon}$ from $\operatorname{Aut}(A)$ to $\operatorname{Picgr}^{\Delta}(R)$. Note that we have $\operatorname{Cen}(A)_{0} \simeq R$, since $A$ is $\varepsilon$-central (cf. 33). Therefore, by 9 (3), we obtain an exact sequence

$$
1 \longrightarrow R^{*} \longrightarrow A_{0}^{*} \stackrel{i_{A}^{0}}{\longrightarrow} \operatorname{Aut}(A) \stackrel{\psi_{A}^{\varepsilon}}{\longrightarrow} \operatorname{Picgr}^{\Delta}(R)
$$

with

$$
\operatorname{Im} \psi_{A}^{\varepsilon}=\left\{\operatorname{cl}(L) \mid A \otimes_{R} L \simeq A \text { as graded left } A \text {-modules }\right\} .
$$

Also, by 38, we obtain an exact sequence

$$
1 \longrightarrow R^{*} \longrightarrow \tilde{h} U(A) \stackrel{i_{A}^{\varepsilon}}{\longrightarrow} \operatorname{Aut}(A) \stackrel{\varphi_{A}^{\varepsilon}}{\longrightarrow} \operatorname{Pic}(R)
$$

where $\varphi_{A}^{\varepsilon}$ is the composite $\operatorname{Aut}(A) \stackrel{\psi_{A}^{\varepsilon}}{\longrightarrow} \operatorname{Picgr}^{\Delta}(R) \stackrel{\text { can. }}{\longrightarrow} \operatorname{Pic}(R)$. 


\title{
References
}

[1] Auslander, M. and Goldman, O., The Brauer group of a commutative ring, Trans. Amer. Math. Soc. 97 (1960), 367-409.

[2] Bass, H., Lecture on topics in algebraic $K$-theory, Tata Inst. Fund. Res. Lectures on Math. and Phys., Bombay, 1967.

[ 3 ] Bass, H., Clifford algebra and spinor norms over a commutative ring, Amer. J. Math. 96 (1974), 156-206.

[4] Bourbaki, N., Éléments de mathématique, Algèbre, Caps. I-III, English translation, AddisonWesley Publishing Co., Reading Mass., 1974; Chap. VIII, Hermann, Paris, 1958.

[5] Bourbaki, N., Éléments de mathématique, Algèbre commutative, Chaps, 1 à 4, Masson \& Cie, Paris, 1985.

[6] Childs, L. N., Garfinkel, G. and Orzech, M., The Brauer group of graded Azumaya algebras, Trans. Amer. Math. Soc., 175 (1973), 299-326.

[7] Long, F. W., A generalization of the Brauer group of graded algebras, Proc. London Math. Soc., 29 (1974), 237-256.

[ 8 ] Mitchell, B., Theory of categories, Pure and Applied Mathematics, Vol. XVII, Academic Press, New York-London, 1965.

[9] Orzech, M., On the Brauer group of algebras having a grading and an action, Can. J. Math., XXVIII, No. 3, 1976, 533-552.

[10] Rosenberg, A. and Zelinsky, D., Automorphisms of separable algebras, Pacific J. Math. 11 (1961), 1109-1117.

[11] Small, C., The Brauer-Wall group of a commutative ring, Trans. Amer. Math. Soc. 156 (1971), 455-491.

\author{
Mathematical Institute \\ Tohoku University \\ Sendai $980-8578$ \\ Japan \\ e-mail address: ikai@math.tohoku.ac.jp
}

